\title{
The Optimal Age of Vaccination Against Dengue in Brazil based on Serotype-Specific Forces of Infection derived from Serological Data
}

\author{
Sandra B. Maier ${ }^{1}$, Eduardo Massad ${ }^{2,3,5}$, Marcos Amaku ${ }^{3}$, Marcelo N. \\ Burattini $^{3,4}$ and David Greenhalgh ${ }^{1}$, \\ ${ }^{1}$ Department of Mathematics and Statistics, University of Strathclyde, Glasgow G1 1XH, \\ U.K., \\ ${ }^{2}$ School of Applied Mathematics, Fundacao Getulio Vargas, Rio de Janeiro, Brazil, \\ ${ }^{3}$ LIM01-Hospital de Clínicas, Faculdade de Medicina, Universidade de São Paulo, São \\ Paulo, SP, Brazil, \\ ${ }^{4}$ Hospital São Paulo, Escola Paulista de Medicina, Universidade Federal de São Paulo, São \\ Paulo, SP, Brazil, \\ ${ }^{5}$ London School of Hygiene and Tropical Medicine, London, U.K.
}

\begin{abstract}
In this paper we study a single serotype transmission model of dengue to determine the optimal vaccination age for Dengvaxia. The transmission dynamics are modelled with an age-dependent force of infection. The force of infection for each serotype is derived from the serological profile of dengue in Brazil without serotype distinction and from serotype-specific reported cases. The risk due to an infection is measured by the probability of requiring hospitalisation based on Brazilian Ministry of Health data.

The optimal vaccination age is determined for any number and combination of the four distinct dengue virus serotypes DENv1-4. The lifetime expected risk is adapted to include antibody dependent enhancement (ADE) and permanent cross-immunity after two heterologous infections. The risk is assumed to be serostatus-dependent. The optimal vaccination age is computed for constant, serostatus-specific vaccine efficacies. Additionally, the vaccination age is restricted to conform to the licence of Dengvaxia in Brazil and the achievable and minimal lifetime expected risks are compared.

The optimal vaccination age obtained for the risk of hospitalisation varies significantly with the assumptions relating to ADE and cross-immunity. Risk-free primary infections leads to higher optimal vaccination ages, as do asymptomatic third and fourth infections. Sometimes vaccination is not recommended at all, for example for any endemic area with a single serotype if primary infections are risk-free. Restricting the vaccination age to Dengvaxia licensed ages mostly leads to only a slightly higher lifetime expected risk and the vaccine should be administered as close as possible to the optimal vaccination age.
\end{abstract}

Keywords: Dengue, Vaccination, Optimal Vaccination Age, Age-Structured Mathematical Model, Serological Data, Hospitalisation Risk

\section{Introduction}

There are four distinct dengue virus serotypes DENv1-4 which are transmitted through mosquitoes. The disease is endemic in over 100 countries and causes an estimated 400 million infections globally each year $[1,2]$. However, the majority of these infections are asymptomatic and go unreported. Roughly $25 \%$ of dengue cases are symptomatic with the 
severity of symptoms varying significantly from a mild fever to plasma leakage and organ failure [2, 3]. 20,000 deaths occur annually due to severe dengue (SD).

Brazil has the highest reported number of dengue infections each year and the incidence and burden of dengue is still increasing in the country [4]. The primary vector of dengue in Brazil is the Aedes aegypti mosquito. Extensive eradication programmes led to Brazil being declared free of the mosquito in the 1950s, but after this initial success the relaxation of the programmes quickly led to a reintroduction of the mosquito which can now be found in nearly $80 \%$ of the country $[5,6]$. The first dengue epidemic after the reintroduction of the Aedes aegypti in 1976 was due to DENv1 and DENv4 in 1980-1981. For the following years DENv1 was the main serotype in circulation. When DENv2 was introduced to Brazil in 1990 the first cases of SD were observed. However, since then the number of SD cases has increased significantly $[1,7]$. Currently all dengue virus serotypes coexist in Brazil with DENv1 being the most prevalent serotype. Outbreaks occur with spatial-temporal variations and are recorded throughout the year, but particularly during the rainy season.

The coexistence of the four dengue virus serotypes leads to the possibility of sequential infections with different serotypes and to potential cross-reactions. An initial infection with any one of the serotypes results in life-long immunity to that serotype and confers short-term protection against the remaining serotypes [8]. However, once the antibodies decline and this cross-protection wanes a secondary, heterologous infection is believed to cause more severe symptoms due to antibody dependent enhancement (ADE) [9]. ADE is caused during a secondary infection by serotype-specific antibodies, which were developed upon exposure to the first serotype, binding on to the heterotypic virus without neutralizing it. The active virus can then enter its target cells and replicate, resulting in a higher virulence and an increased risk of SD. This mechanism is believed to be the main cause of SD since most of these cases are observed either in secondary infections or in infants with low levels of maternal antibodies [9-11]. Post-secondary infections are hardly ever recorded, so that it is theorised that two heterologous infections confer permanent cross-immunity or that third and fourth 
infections are asymptomatic $[12,13]$.

The complex interactions between the serotypes pose a challenge when developing a vaccine against dengue. However, after 20 years of development the first dengue vaccine, Dengvaxia, has been licensed for use in individuals aged either 9 to 45 years or 9 to 60 years in several countries including Brazil $[14,15]$. Unfortunately, despite the demonstrated safety and efficacy of Dengvaxia in phase three clinical trials [16, 17], concerns were raised regarding its application in seronegative recipients [18-20]. Initially the recommendation was to vaccinate individuals aged at least 9 years in highly endemic settings only [3], but with more data becoming available from the long-term follow-up phase of the clinical trials the recommendations regarding the use of Dengvaxia have been revised so as to vaccinate only individuals who have had a prior dengue infection [21, 22].

The aim of this paper is to develop a single serotype model of the transmission dynamics of dengue that can be used to capture the complex interactions between the different serotypes when vaccination is considered without explicitly modelling them. This model is then used to compute the lifetime expected risk due to dengue infections and to determine the optimal vaccination age which minimises this risk. The use of a single serotype model has the advantage of being easier to investigate both analytically and computationally than multi-serotype models. Meanwhile the effect of the different serotype interactions can be explored by adapting the function describing the risk of dengue. Serotype-specific forces of infection are determined using serological data from Brazil. Additionally, the risk is based on the probability of hospitalisation derived from pre-vaccine data collected in Brazil and it is assumed that the risk is serostatus-dependent according to the long-term follow-up data of clinical trials $[3,21,23]$.

\section{Vaccination Model}

The optimal age of vaccination against dengue can be defined as the vaccination age which leads to the minimal lifetime expected risk as done for measles by Hethcote [24]. The 
computation of the lifetime expected risk requires the transmission dynamics of the disease to be modelled mathematically. A specific vaccination strategy can be incorporated in the model through matching conditions. For the sake of simplicity a single serotype model is used and while many of the model parameters are serotype independent some parameters such as the vaccine related parameters in the matching conditions and the force of infection are serotype specific. The serotype interactions, while not modelled explicitly, can be incorporated in the lifetime expected risk.

Maier et al. [25] introduced a single serotype model describing the transmission dynamics of dengue for a constant force of infection and a constant human death rate. This model can be adapted to describe the dynamics in the case of an age-dependent force of infection $\lambda_{H}(a, t)$ when the age-dependence is assumed to arise from the mosquito biting rate $q(a)$ varying with age of the human, and for an age-dependent death function $\mu_{H}(a)$ for the human population. If the assumptions relating to all other aspects are as described by Maier et al. [25] and the forces of infection in the respective human and mosquito populations are given by

$$
\lambda(a, t)=q(a) \frac{b I_{M}(t)}{N_{H}} \quad \text { and } \quad \lambda_{M}(t)=c \int_{0}^{\infty} q(a) \frac{I_{H}(a, t)}{N_{H}} d a
$$

the resulting transmission model is

$$
\begin{aligned}
\frac{\partial U_{H}}{\partial a}+\frac{\partial U_{H}}{\partial t} & =-\lambda(a, t) C(a) U_{H}(a, t)-\mu_{H}(a) U_{H}(a, t), \\
\frac{\partial I_{H}}{\partial a}+\frac{\partial I_{H}}{\partial t} & =\lambda(a, t) C(a) U_{H}(a, t)-\left(\mu_{H}(a)+\gamma_{H}\right) I_{H}(a, t), \\
\frac{\partial R_{H}}{\partial a}+\frac{\partial R_{H}}{\partial t} & =\gamma_{H} I_{H}(a, t)-\mu_{H}(a) R_{H}(a, t), \\
\frac{\partial N_{H}}{\partial a}+\frac{\partial N_{H}}{\partial t} & =-\mu_{H}(a) N_{H}(a, t), \\
N_{H}(a, t) & =U_{H}(a, t)+I_{H}(a, t)+R_{H}(a, t)
\end{aligned}
$$




$$
\begin{aligned}
\frac{d S_{M}}{d t} & =-\lambda_{M}(t) S_{M}(t)-\mu_{M} S_{M}(t)+\mu_{M} N_{M}, \\
\frac{d L_{M}}{d t} & =\lambda_{M}(t) S_{M}(t)-e^{-\mu_{M} \tau} \lambda_{M}(t-\tau) S_{M}(t-\tau)-\mu_{M} L_{M}(t), \\
\frac{d I_{M}}{d t} & =e^{-\mu_{M} \tau} \lambda_{M}(t-\tau) S_{M}(t-\tau)-\mu_{M} I_{M}(t), \\
N_{M}(t) & =S_{M}(t)+L_{M}(t)+I_{M}(t),
\end{aligned}
$$

where $U_{H}(a, t), I_{H}(a, t)$, and $R_{H}(a, t)$ describe the age-density of unaffected, infected and recovered humans respectively, and $S_{M}(t), L_{M}(t)$, and $I_{M}(t)$ the total number of susceptible, exposed, and infectious mosquitoes. Note that unaffected humans comprise both individuals protected by maternal antibodies and those susceptible to infection. The initial conditions and matching conditions for a three-dose vaccination strategy are given by

$$
\begin{aligned}
& U_{H}(a, 0)=U_{H, 0}(a), I_{H}(a, t)=I_{H, 0}(a) \text { for } t \in[-\tau, 0], R_{H}(a, 0)=R_{H, 0}(a) \\
& \text { and } N_{H, 0}(a, 0)=N_{H, 0}(a)=U_{H, 0}(a)+I_{H, 0}(a)+R_{H, 0}(a) ; \\
& U_{H}(0, t)=\mu_{H} N_{H}, I_{H}(0, t)=0, R_{H}(0, t)=0 \text { and } N_{H}(0, t)=\mu_{H} N_{H} ; \\
& S_{M}(t)=S_{M, 0} \text { for } t \in[-\tau, 0], L_{M}(0)=L_{M, 0}, I_{M}(0)=I_{M, 0} \\
& \text { and } N_{M}(0)=N_{M, 0}=S_{M, 0}+L_{M, 0}+I_{M, 0} .
\end{aligned}
$$

Moreover for each vaccination dose $i$ and corresponding vaccination age $A_{i}$

$$
\lim _{a \rightarrow A_{i}^{+}} U_{H}(a, t)=\left(1-V_{i} C\left(A_{i}\right)\right) \lim _{a \rightarrow A_{i}^{-}} U_{H}(a, t)
$$

The three doses are assumed to be administered 6 months apart based on the licence restriction of Dengvaxia. The death function $\mu_{H}(a)$ and corresponding survival probability $\pi(a)$ are assumed to be step-death functions with expected lifetime $L=73.8$ years [26], i.e.

$$
\mu_{H}(a)=\left\{\begin{array}{ll}
0, & 0 \leq a<L, \\
\infty, & L \leq a<\infty,
\end{array} \quad \text { and } \quad \pi(a)= \begin{cases}1, & 0 \leq a<L \\
0, & L \leq a<\infty\end{cases}\right.
$$

The parameters and functions used are summarised in Table 1. 
Table 1: Description of model parameters and their values used in calculations where appropriate.

\begin{tabular}{|c|c|c|c|}
\hline & Significance & Value & Source \\
\hline$\mu_{H}(a)$ & step-death function for the human population & c.f. Equation (4) & \\
\hline$\pi(a)$ & survival probability for the human population & c.f. Equation (4) & \\
\hline$q(a)$ & $\begin{array}{l}\text { age-dependent rate at which humans are bitten by } \\
\text { mosquitoes }\end{array}$ & c.f. Equation (20) & \\
\hline$b$ & $\begin{array}{l}\text { transmission probability from infected mosquito to } \\
\text { susceptible human }\end{array}$ & 0.6 & {$[27]$} \\
\hline$c$ & $\begin{array}{l}\text { transmission probability from infected human to } \\
\text { susceptible mosquito }\end{array}$ & 1 & {$[27]$} \\
\hline$m$ & number of mosquitoes per human $\left(m=\frac{N_{M}}{N_{H}}\right)$ & 1.5 & {$[28]$} \\
\hline$\gamma_{H}$ & per capita recovery rate of humans & 51.135 year $^{-1}$ & {$[27]$} \\
\hline$\mu_{M}$ & natural per capita death rate of mosquitoes & 9.131 year $^{-1}$ & {$[27]$} \\
\hline$\tau$ & extrinsic incubation period & 0.0192 years & {$[27]$} \\
\hline$L$ & expected lifetime of humans in Brazil & 73.8 years & {$[26]$} \\
\hline$\delta_{i}(a)$ & $\begin{array}{l}\text { the age-related serotype specific rate of } \\
\text { loss of maternal antibodies }\end{array}$ & & {$[29]$} \\
\hline$C_{i}(a)$ & $\begin{array}{l}\text { the age-dependent serotype-specific seroconversion } \\
\text { rate corresponding to loss of maternal antibodies }\end{array}$ & $1-e^{-\int_{0}^{a} \delta_{i}(\xi) d \xi}$ & {$[24]$} \\
\hline$A_{i}$ & $\begin{array}{l}\text { the vaccination age for each of the three vaccination } \\
\text { doses } i=1,2,3\end{array}$ & $0-L$ years & \\
\hline$V_{i}$ & $\begin{array}{l}\text { the proportion vaccinated for the three vaccination } \\
\text { doses } i=1,2,3 \text { where } V_{i}=1-(1-\text { eff })^{1 / 3} \text { depends } \\
\text { on the serotype-specific vaccine efficacy eff }\end{array}$ & c.f. Table 3 & \\
\hline
\end{tabular}

\subsection{Steady-State Dynamics}

At the steady-state Equations (2) and (3) can be solved to obtain the steady-state agedensities of unaffected, infected and recovered humans as well as the steady-state force of infection $\lambda(a)$ similarly to the case of a constant force of infection [25]. This can be done both in the presence and in the absence of a vaccination campaign.

Let $U_{H}(a)$ denote the equilibrium density with respect to age of unaffected individuals of age $a$ and $N_{H}(a)$ the density with respect to age of the total number of individuals of age $a$, the fraction of the population of age $a$ who are unaffected is then given by:

$$
u(a)=U_{H}(a) / N_{H}(a)
$$

We similarly define $i(a)$ and $r(a)$ to be the fractions of the population of age $a$ who are 
infected and immune and note that $r(a)=1-u(a)-i(a)$. Then Equation (2) reduces to the system

$$
\begin{aligned}
& \frac{d u}{d a}=-\lambda(a) C(a) u(a), \\
& \frac{d i}{d a}=\lambda(a) C(a) u(a)-\gamma_{H} i(a),
\end{aligned}
$$

with initial conditions $u(0)=1$ and $i(0)=0$, and if vaccination takes place the additional matching conditions $\lim _{a \rightarrow A_{i}^{+}} u(a)=\left(1-V_{i} C\left(A_{i}\right)\right) \lim _{a \rightarrow A_{i}^{-}} u(a)$ for $i=1,2,3$. These ordinary differential equations can analytically be solved to obtain

$$
u(a)= \begin{cases}e^{-\int_{0}^{a} \lambda(s) C(s) d s}, & 0 \leq a<A_{1}, \\ \left(1-V_{1} C\left(A_{1}\right)\right) e^{-\int_{0}^{a} \lambda(s) C(s) d s}, & A_{1} \leq a<A_{2}, \\ \left(1-V_{1} C\left(A_{1}\right)\right)\left(1-V_{2} C\left(A_{2}\right)\right) e^{-\int_{0}^{a} \lambda(s) C(s) d s}, & A_{2} \leq a<A_{3}, \\ \left(1-V_{1} C\left(A_{1}\right)\right)\left(1-V_{2} C\left(A_{2}\right)\right)\left(1-V_{3} C\left(A_{3}\right)\right) e^{-\int_{0}^{a} \lambda(s) C(s) d s}, & A_{3} \leq a<\infty,\end{cases}
$$

for the steady-state age-density of unaffected, and

$$
i(a)=e^{-\gamma_{H} a} \int_{0}^{a} \lambda(s) C(s) u(s) e^{\gamma_{H} s} d s
$$

for the steady-state proportion of humans of age $a$ who are infected. Additionally, from the matching conditions one obtains the fraction of successfully vaccinated individuals $v(a)$ as

$$
v(a)= \begin{cases}0, & 0 \leq a \leq A_{1}, \\ V_{1} C\left(A_{1}\right) u\left(A_{1}^{-}\right), & A_{1}<a \leq A_{2}, \\ V_{1} C\left(A_{1}\right) u\left(A_{1}^{-}\right)+V_{2} C\left(A_{2}\right) u\left(A_{2}^{-}\right), & A_{2}<a \leq A_{3}, \\ V_{1} C\left(A_{1}\right) u\left(A_{1}^{-}\right)+V_{2} C\left(A_{2}\right) u\left(A_{2}^{-}\right)+V_{3} C\left(A_{3}\right) u\left(A_{3}^{-}\right), & A_{3}<a<\infty .\end{cases}
$$

In the absence of vaccination, i.e. before the introduction of a vaccine, the corresponding 
steady-state fractions can be denoted by $u_{0}(a)$ and $i_{0}(a)$ are given by

$$
\begin{aligned}
u_{0}(a) & =e^{-\int_{0}^{a} \lambda_{0}(s) C(s) d s} \\
& \text { and } \quad i_{0}(a)=e^{-\gamma_{H} a} \int_{0}^{a} \lambda_{0}(s) C(s) u_{0}(s) e^{\gamma_{H} s} d s
\end{aligned}
$$

where $\lambda_{0}(a)$ is the pre-vaccine steady-state force of infection and trivially $v_{0}(a)=0$.

By solving the steady-state dynamics of the mosquito equations as described for a constant force of infection [25] one obtains an expression for the number of infectious mosquitoes at the steady-state. This can then be substituted into $\lambda(a)$ as given in Equation (1) to obtain

$$
\lambda(a)=q(a) m b e^{-\mu_{M} \tau} \frac{\frac{c}{L} \int_{0}^{\infty} q(s) i(s) \pi(s) d s}{\mu_{M}+\frac{c}{L} \int_{0}^{\infty} q(s) i(s) \pi(s) d s}
$$

for a given vaccination strategy, or

$$
\lambda_{0}(a)=q(a) m b e^{-\mu_{M} \tau} \frac{\frac{c}{L} \int_{0}^{\infty} q(s) i_{0}(s) \pi(s) d s}{\mu_{M}+\frac{c}{L} \int_{0}^{\infty} q(s) i_{0}(s) \pi(s) d s}
$$

before a vaccination campaign is started. If the pre-vaccine force of infection $\lambda_{0}(a)$ is known the steady-state force of infection for a given vaccination strategy can therefore be computed as

$$
\lambda(a)=\lambda_{0}(a) \frac{\int_{0}^{\infty} q(s) i(s) \pi(s) d s}{1+\frac{c}{\mu_{M}} \int_{0}^{\infty} q(s) i(s) \pi(s) d s} \frac{1+\frac{c}{\mu_{M}} \int_{0}^{\infty} q(s) i_{0}(s) \pi(s) d s}{\int_{0}^{\infty} q(s) i_{0}(s) \pi(s) d s}
$$

\section{Serotype-Specific Forces of Infection from Serological Data}

The force of infection, $\lambda_{0}(a)$, for all serotypes combined in the absence of a vaccination campaign can be determined from serological data as has been shown by Farrington [30] for other diseases. This is achieved by fitting $s_{0}^{+}(a)$, a function describing the proportion of seropositives at age $a$ in the absence of vaccination, to the serological data and applying the catalytic method to obtain $\lambda_{0}(a)=\frac{d s_{0}^{+}(a)}{d a}\left(1-s_{0}^{+}(a)\right)^{-1}$. According to Farrington [30] a consistent age-dependent force of infection needs to be non-negative for all ages, and low at birth since newborns are protected by maternal antibodies. The proportion of seropositives 
given by $s_{0}^{+}(a)=1-e^{-\Lambda_{0}(a)}$ with

$$
\Lambda_{0}(a)=\int_{0}^{a} \lambda_{0}(s) d s=k_{3} a-\frac{k_{1}}{k_{2}} a e^{-k_{2} a}-\frac{1}{k_{2}}\left(\frac{k_{1}}{k_{2}}-k_{3}\right)\left(e^{-k_{2} a}-1\right)
$$

results in the consistent force of infection

$$
\lambda_{0}(a)=\left(k_{1} a-k_{3}\right) e^{-k_{2} a}+k_{3} .
$$

This definition of the proportion of seropositives includes only individuals that are seropositive due to an infection but not those that are passively immune. However if serological data records the proportion of seropositives at young ages it can be assumed that some of the early data will be influenced by maternal antibodies as serological tests cannot distinguish between maternal antibodies and antibodies caused by a prior dengue infection. In this case the data can be fitted to the age-dependent fraction of seropositives due to both infection and maternal antibodies in order to increase the accuracy of the fit.

We assume that the number of individuals protected by maternal antibodies decays at a constant rate per unit time [29]. We separate the unaffected individuals of age $a$ at time $t$ into those protected by maternal antibodies and those susceptible. We denote the age densities of the number of humans protected by maternal antibodies and of those who are susceptible at time $t$ by $M_{H}(a, t)$ and $S_{H}(a, t)$ respectively. Then in Equation (2) the equation for the age density of unaffected humans is replaced by

and

$$
\begin{aligned}
\frac{\partial M_{H}}{\partial a}+\frac{\partial M_{H}}{\partial t} & =-\delta M_{H}(a, t)-\mu(a) M_{H}(a, t), \\
\frac{\partial S_{H}}{\partial a}+\frac{\partial S_{H}}{\partial t} & =\delta M_{H}(a, t)-(\lambda(a)+\mu(a)) S_{H}(a, t),
\end{aligned}
$$

with $M_{H}(0, t)=\mu_{H} / L, S_{H}(0, t)=0$ for all $t$, and given initial conditions $M_{H}(a, 0)=M_{H, 0}(a)$ and $S_{H}(a, 0)=S_{H, 0}(a)$. Here $\delta$ is the constant rate of decay of maternal antibodies so $1 / \delta$ is the average period for which an individual is protected by maternal antibodies.

If we define $m(a)$ to be the equilibrium fraction of individuals of age $a$ who are protected 
by maternal antibodies, then

$$
\begin{aligned}
m(a) & =e^{-\delta a}, \\
\text { and } \quad \frac{d s}{d a} & =\delta m-\lambda(a) s,
\end{aligned}
$$

with initial conditions $m(0)=1$ and $s(0)=0$. We deduce that

and

$$
\frac{d m}{d a}=-\delta m
$$

$$
s(a)=e^{-\Lambda(a)}-e^{-\delta a}+\int_{0}^{a} \lambda(s) e^{-\delta s-(\Lambda(a)-\Lambda(s)) d s} .
$$

Here $\Lambda(a)=\int_{0}^{a} \lambda(s) d s$.

Hence $\bar{s}_{0}^{+}(a)$, the equilibrium fraction of individuals of age $a$ who are seropositive (in other words either protected by maternal antibodies, infected or immune), is given by

$$
\begin{aligned}
{\overline{s_{0}}}^{+}(a) & =1-s_{0}(a), \\
& =1-e^{-\Lambda_{0}(a)}+e^{-\delta a}-\int_{0}^{a}\left(\left(k_{1} s-k_{3}\right) e^{-k_{2} s}+k_{3}\right) e^{-\delta s-\left(\Lambda_{0}(a)-\Lambda_{0}(s)\right)} d s .
\end{aligned}
$$

However, for the traditional catalytic method [30] the effect of maternal antibodies is ignored and only seropositives due to infection $s_{0}^{+}(a)$ are considered and the force of infection is therefore derived as Equation (13) from the fitted parameters. The rate of loss of maternal antibodies $\delta$ can either be taken from literature or fitted as an additional parameter.

The serological data which was available to us [31] is not serotype-specific but rather considers dengue as a whole, so that the force of infection resulting from the catalytic method is also for all serotypes combined. The proportion of seropositives for ages between 1 and 20 years in Brazil was recorded as given in Figure 1. We fitted the data to a function of the form Equation (14) with $\lambda(a)=\lambda_{0}(a)$ to correct for the effect of maternal antibodies. $\delta$ was fitted as an additional parameter since values given in the literature vary and depend on the specific serotype considered [29, 32, 33]. 


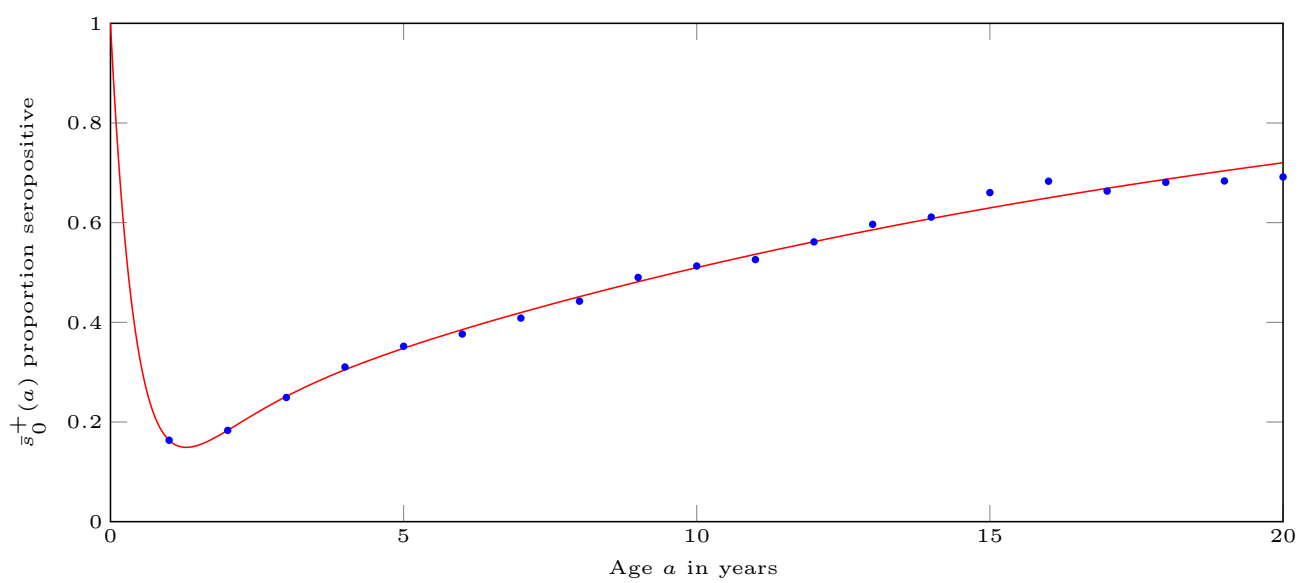

Figure 1: Serological data (data points) from [31] and the fitted function of type $\bar{s}_{0}^{+}(a)$ given by Equation (14) (solid line) where $\delta$ was fitted as an additional parameter.

The estimate we obtained for $\lambda_{0}(a)$ is

$$
\lambda_{0}(a)=\left((0.3086 a-0.0561) e^{-1.1441 a}+0.0561\right) \mathrm{year}^{-1}
$$

and our estimate for $\delta$, the rate at which maternal antibodies decay, is 2.3350 year $^{-1}$, leading to an average period of protection by maternal antibodies of 0.4283 years.

We now want to use the estimate of $\lambda_{0}(a)$ to determine the force of infection for each of the four serotypes separately. Considering Equation (11) for each serotype implies

$$
\lambda_{0}^{i}(a)=\xi_{i} q(a)
$$

where $\xi_{i}(i=1,2,3,4)$ is a constant. Further the overall force of infection from dengue is the sum of the separate forces of infection, i.e.

$$
\begin{aligned}
\lambda_{0}(a) & =\left(\xi_{1}+\xi_{2}+\xi_{3}+\xi_{4}\right) q(a) \\
& =\xi_{T} q(a) .
\end{aligned}
$$

Using Equations (16) and (17) and approximating the ratio $\xi_{1}: \xi_{2}: \xi_{3}: \xi_{4}$ by the ratio of the number of dengue cases caused by each serotype $n_{i}(i=1,2,3,4)$ as described in Appendix A 


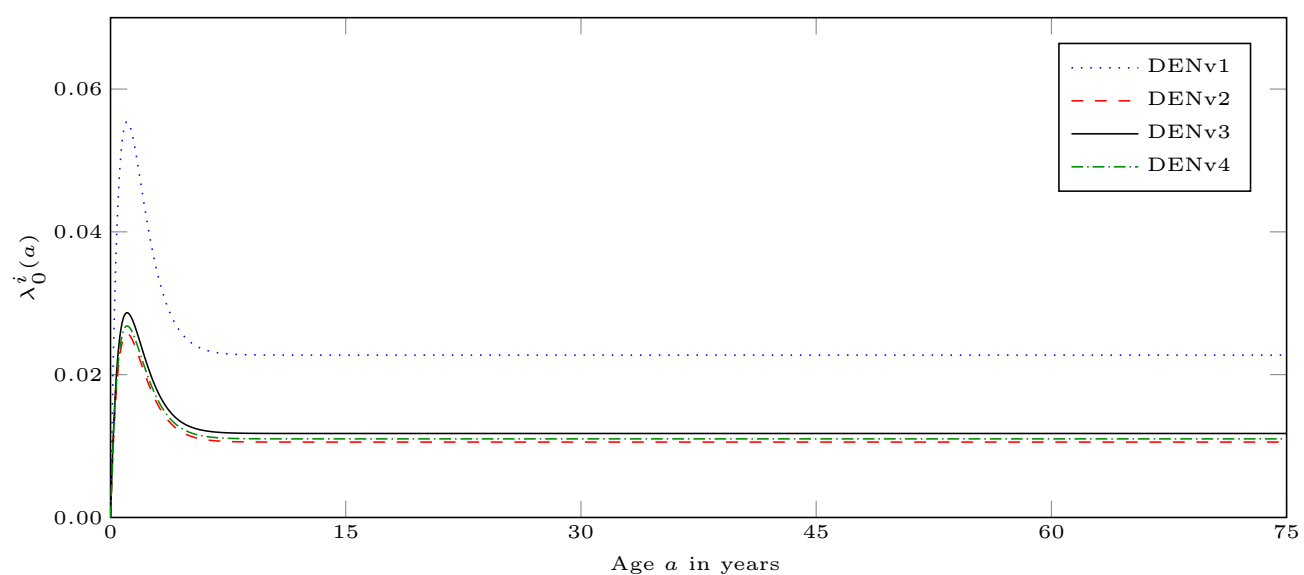

Figure 2: Serotype specific forces of infection before the introduction of a vaccination campaign derived from seroprevalence data and serotype specific case report data.

we obtain

$$
\begin{aligned}
\lambda_{0}^{i}(a) & =\frac{\xi_{i}}{\xi_{1}+\xi_{2}+\xi_{3}+\xi_{4}} \lambda_{0}(a) \\
& =\frac{n_{i}}{n_{1}+n_{2}+n_{3}+n_{4}} \lambda_{0}(a) .
\end{aligned}
$$

The serotype-specific forces of infection based on case report data collected by the Brazilian Ministry of Health (SINAN) between 2000 and 2014 are presented in Figure 2. While the SINAN case report data are generally not serotyped, the serotype in a relatively small number of cases is determined. Specific serotyping of dengue virus started in 2006 and we have data for the period 2006-2014 [34]. From these small samples it is possible to infer the number of cases for each serotype [35]. From the case report data we can then see that DENv1 caused most infections between 2000 and 2014, followed by DENv3, DENv4 and lastly DENv2. This is reflected in the forces of infection presented in Figure 2.

Note that by substituting Equation (17) into Equation (11) we can deduce

$$
1=\frac{m b e^{-\mu_{M} \tau} \frac{c}{L} \int_{0}^{\infty} \lambda_{0}(a) i_{0}(a) \pi(a) d a}{\xi_{T}\left(\mu_{M} \xi_{T}+\frac{c}{L} \int_{0}^{\infty} \lambda_{0}(a) i_{0}(a) \pi(a) d a\right)} .
$$

Assuming that an exposed unaffected individual will seroconvert unless they are protected by maternal antibodies, i.e. $C(a)=1-e^{\delta a}$ (Hethcote [24]), and using $\lambda_{0}(a)$ as given 
in Equation (15) we can numerically determine the unique strictly positive $\xi_{T}$ that solves Equation (19) and thus deduce the biting rate from Equation (17) as

$$
q(a)=\left((162.1207 a-29.4558) e^{-1.1441 a}+29.4558\right) \text { year }^{-1}
$$

\section{Risk of Infection}

The lifetime expected risk of dengue for the model with a constant force of infection was

derived in [25] where the definition for the lifetime expected risk of measles by Hethcote [24] was adapted to allow for the coexistence of several serotypes. Additionally the survival probability $\pi(a)$ was included in the definition to prevent the overestimation of the risk associated with infections at older ages. However, the risk was not assumed to depend on whether an individual was seropositive due to a previous infection or due to vaccination. Using a similar notation as in [25] but differentiating between seropositivity due to vaccination or infection the expected risk from infection with serotype $i$ at age $a$ is denoted by

$$
\begin{aligned}
E_{i}(a)= & P_{i \bar{j} \bar{k} \bar{l}}(a) R_{i \bar{j} \bar{k} \bar{l}}(a)+P_{i j \bar{k} \bar{l}}(a) R_{i j \bar{k} \bar{l}}(a)+P_{i \bar{j} k \bar{l}}(a) R_{i \bar{j} k \bar{l}}(a)+P_{i \bar{j} \bar{k} l}(a) R_{i \bar{j} \bar{k} l}(a) \\
& +P_{i j_{*} \bar{k} l}(a) R_{i j_{*} \bar{k} \bar{l}}(a)+P_{i \bar{j} k_{*} \bar{l}}(a) R_{i \bar{j} k_{*} \bar{l}}(a)+P_{i \bar{j} \bar{k} l_{*}}(a) R_{i \bar{j} \bar{k} l_{*}}(a)+P_{i j k \bar{l}}(a) R_{i j k \bar{l}}(a) \\
& +P_{i j \bar{k} l}(a) R_{i j \bar{k} l}(a)+P_{i \bar{j} k l}(a) R_{i \bar{j} k l}(a)+P_{i j_{*} k_{*} \bar{l}}(a) R_{i j_{*} k_{*} \bar{l}}(a)+P_{i j_{*} \bar{k} l_{*}}(a) R_{i j_{*} \bar{k} l_{*}}(a) \\
& +P_{i \bar{j} k_{*} l_{*}}(a) R_{i \bar{j} k_{*} l_{*}}(a)+P_{i j k_{*} \bar{l}}(a) R_{i j k_{*} \bar{l}}(a)+P_{i j \bar{k} l_{*}}(a) R_{i j \bar{k} l_{*}}(a)+P_{i j_{*} k \bar{l}}(a) R_{i j_{*} k \bar{l}}(a) \\
& +P_{i j_{*} \bar{k} l}(a) R_{i j_{*} \bar{k} l}(a)+P_{i \bar{j} k l_{*}}(a) R_{i \bar{j} k l_{*}}(a)+P_{i \bar{j} k_{*} l}(a) R_{i \bar{j} k_{*} l}(a)+P_{i j k l}(a) R_{i j k l}(a) \\
& +P_{i j k l_{*}}(a) R_{i j k l_{*}}(a)+P_{i j k_{*} l}(a) R_{i j k_{*} l}(a)+P_{i j_{*} k l}(a) R_{i j_{*} k l}(a)+P_{i j k_{*} l_{*}}(a) R_{i j k_{*} l_{*}}(a) \\
& +P_{i j_{*} k l_{*}}(a) R_{i j_{*} k l_{*}}(a)+P_{i j_{*} k_{*} l}(a) R_{i j_{*} k_{*} l}(a)+P_{i j_{*} k_{*} l_{*}}(a) R_{i j_{*} k_{*} l_{*}}(a),
\end{aligned}
$$

where $P_{i j k_{*}}(a)$, for example, is the probability of an individual who has had a prior natural infection with serotype $j$ (subscript $j$ ), has been successfully vaccinated against serotype $k$ (subscript $k_{*}$ ) and has never been infected with or successfully vaccinated against serotype $l$ (subscript $\bar{l}$ ) to get infected by serotype $i$ at age $a . R_{i j k_{*}}(a)$ is the risk associated with such an infection and vaccination history. The remaining functions are defined similarly. If there are less than four serotypes in circulation both the probability of infection with a non-existent serotype and the associated risk are interpreted as zero. The lifetime expected risk is then 
given by

$$
E=\int_{0}^{\infty}\left(\sum_{i=1}^{4} E_{i}(a)\right) \pi(a) d a
$$

The infection probabilities can be calculated similarly to [25] from the steady-state agedensities and forces of infection for each of the serotypes. For serotype $i$ the fractions of unaffected and vaccinated are given by $u_{i}(a)$ and $v_{i}(a)$ and therefore the probability of having had a natural infection with serotype $i$ by age $a$ is $1-\left(u_{i}(a)+v_{i}(a)\right)$. We denote the age-dependent force of infection due to serotype $i$ by $\lambda_{i}(a)$ which can be computed from Equation (12) for a specific vaccination strategy. We further take $C_{i}(a)=1-e^{-\int_{0}^{a} \delta_{i}(s) d s}$, where $\delta_{i}(a)$ is the piecewise linear age-dependent rate of loss of maternal antibodies for serotype $i$ based on data taken from van Panhuis et al. [29]. Then, for example,

$$
P_{i j k_{*} \bar{l}}(a)=\lambda_{i}(a) C_{i}(a) u_{i}(a)\left(1-\left(u_{j}(a)+v_{j}(a)\right)\right) v_{k}(a) u_{l}(a) .
$$

All other probabilities are calculated analogously.

The associated risk functions (e.g. $\left.R_{i j k_{*} l}(a)\right)$ need to be defined according to some measure of risk and incorporate the difference in risk depending on serostatus. We derived the agedependent pre-vaccine risk of being admitted to hospital due to an infection with dengue by fitting a piecewise function to data collected by SINAN which was evaluated to determine the age differences in hospitalisation by Burattini et al. [23]. We assumed that the risk remains constant above the maximum recorded age. The risk function we obtained is

$$
R(a)= \begin{cases}0.09153 a e^{-0.1820 a}, & 0 \leq a<21.3333, \\ 0.02428 e^{0.02362 a}, & 21.3333 \leq a<100, \\ 0.02428 e^{0.02362 \cdot 100}, & 100 \leq a<\infty .\end{cases}
$$

The risk of hospitalisation is therefore highest in children, with a peak at approximately 5.5 years. Adults on the other hand have a relatively low risk of being hospitalised due to dengue but with age the risk increases again, so that at the age of 70 years the risk is approximately 
Table 2: Number of hospitalisations and SD cases according to serostatus during the long-term follow-up of Dengvaxia trials in the vaccine and control group (pooled from [3]).

\begin{tabular}{lcc}
\hline & Control Group & Vaccine Group \\
\hline Seropositive & $26 / 988$ & $16 / 2,027$ \\
Seronegative & $9 / 377$ & $24 / 712$ \\
\hline
\end{tabular}

$66 \%$ of the highest risk. With respect to this high risk at older ages it is important to note that the use of a step-death function and the incorporation of the survival probability in the lifetime expected risk results in a cut-off at $L=73.8$ years. The long-term follow-up of several Dengvaxia trials has shown an increased risk of hospitalisation and SD in seronegative recipients compared to seropositive recipients as shown in Table 2 [3]. We use the data in Table 2 however we note that alternative estimates of the risk of hospitalisation calculated from SINAN data are $10.69 \%$ in individuals who were originally seropositive and $10.42 \%$ in individuals who were originally seronegative [23]. The relative risk depending on serostatus can be included in the risk functions associated with a given infection and vaccination history as outlined in Appendix B.

\section{Results}

We now determine the optimal vaccination age for dengue given a specific vaccination strategy with the aim of minimising the lifetime expected risk of hospitalisation when this risk is serostatus-dependent. Dengvaxia, currently the only licensed vaccine against dengue, is recommended as a three-dose series at 0,6 and 12 months so that we will consider the routine vaccination according to this strategy and refer to the optimal vaccination age as the age at which the first dose should be given. While the vaccine efficacy for Dengvaxia and the risk of breakthrough cases were found to depend on age amongst other factors $[3,16,17]$ it has been questioned whether differences in vaccine efficacy and hospitalisation risk are in fact due to serostatus. Aguiar and Stollenwerk $[36,37]$ argue that the serostatus is the determining factor. Dans et al. [38] query the biological basis for subgroups based on age altogether. We therefore consider the serotype-specific efficacies to be age-independent as 
Table 3: Dengvaxia vaccine efficacies obtained from clinical trials $[3,17]$.

\begin{tabular}{lc}
\hline & Vaccine efficacy \\
\hline DENv1 & $54.7 \%$ \\
DENv2 & $43.0 \%$ \\
DENv3 & $71.6 \%$ \\
DENv4 & $76.9 \%$ \\
\hline Seropositive & $78.2 \%$ \\
Seronegative & $38.1 \%$ \\
\hline
\end{tabular}

presented in Table 3 and pooled data for the risk in seronegative and seropositive recipients as shown in Table 2. Cross-reactions are debated to play an important role in disease severity. Some evidence indicates, for example, that secondary infections are riskier than primary ones due to $\operatorname{ADE}[9,10]$ and it is also assumed that tertiary and quaternary infections rarely occur $[12,13]$. We will therefore compare the effect of primary infections being risky or risk-free, as well as that of symptomatic and asymptomatic post-secondary infections. Note that due to Dengvaxia being an imperfect vaccine it is possible that an infection after vaccination can be a tertiary or quaternary type infection even if not all four serotypes coexist. While Dengvaxia is licensed for the use in individuals aged 9-45 years in Brazil we will initially allow vaccination at any age.

In the following subsections the results are first summarised and then presented for endemic areas with one, two, three and four coexisting serotypes when vaccination is permitted at any age. Subsequently the achievable outcome under licence restrictions is considered. The model parameters used in the computations together with their values and sources are given in Table 1.

\subsection{Serostatus-Dependent Hospitalisation Risk}

The results for all considered scenarios are presented in Table 4. All possible combinations of serotypes were considered under two aspects, namely the effect of ADE (risky or risk-free primary infections) and cross-immunity following two heterologous infections (symptomatic or asymptomatic third and fourth infections). In general it can be seen that assuming all infections to be risky, i.e. symptomatic third and fourth infections and risky primary 
Table 4: Optimal vaccination age $A_{1}$ in months to minimise the lifetime expected risk $E$ for the age-dependent risk of hospitalisation when the risk depends on prior infections and vaccination, i.e. is serostatus-dependent. Vaccine efficacy is serotype-specific as given in Table 3. Risky primary infections, as well as risk-free primary infections are considered. Additionally results for symptomatic and asymptomatic post-secondary infections are presented. Ages outwith the life expectancy are represented as '-'; in these cases vaccination is not recommended.

\begin{tabular}{|c|c|c|c|c|c|c|c|c|}
\hline & \multicolumn{4}{|c|}{$\begin{array}{c}\text { Symptomatic } \\
3^{\text {rd }} \text { and } 4^{\text {th }} \text { infections }\end{array}$} & \multicolumn{4}{|c|}{$\begin{array}{c}\text { Asymptomatic } \\
3^{\text {rd }} \text { and } 4^{\text {th }} \text { infections }\end{array}$} \\
\hline & \multicolumn{2}{|c|}{$1^{\text {st }}$ infection risky } & \multicolumn{2}{|c|}{$1^{\text {st }}$ infection risk-free } & \multicolumn{2}{|c|}{$1^{\text {st }}$ infection risky } & \multicolumn{2}{|c|}{$1^{\text {st }}$ infection risk-free } \\
\hline & $A_{1}$ & $E$ & $A_{1}$ & $E$ & $A_{1}$ & $E$ & $A_{1}$ & $E$ \\
\hline & & $\times 10^{-2}$ & & $\times 10^{-2}$ & & $\times 10^{-2}$ & & $\times 10^{-2}$ \\
\hline DENv1 & 9 & 6.12 & - & 0.00 & - & 12.37 & - & 0.00 \\
\hline DENv2 & 182 & 4.81 & - & 0.00 & - & 9.65 & - & 0.00 \\
\hline DENv3 & 12 & 1.73 & - & 0.00 & 17 & 8.75 & - & 0.00 \\
\hline DENv4 & 32 & 1.50 & - & 0.00 & 56 & 6.83 & - & 0.00 \\
\hline DENv12 & 102 & 9.85 & 589 & 5.09 & 453 & 19.91 & 640 & 14.43 \\
\hline DENv13 & 11 & 7.47 & 445 & 4.77 & 445 & 19.57 & 618 & 14.32 \\
\hline DENv14 & 23 & 7.36 & 449 & 4.39 & 449 & 19.30 & 640 & 13.82 \\
\hline DENv23 & 11 & 6.37 & 667 & 3.68 & 253 & 18.25 & - & 12.86 \\
\hline DENv24 & 48 & 6.21 & 640 & 3.43 & 242 & 17.46 & - & 12.30 \\
\hline DENv34 & 17 & 3.23 & 242 & 2.66 & 56 & 14.41 & 696 & 12.05 \\
\hline DENv123 & 11 & 11.03 & 287 & 8.05 & 384 & 20.79 & 491 & 17.05 \\
\hline DENv124 & 48 & 10.80 & 296 & 7.65 & 384 & 20.72 & 499 & 16.79 \\
\hline DENv134 & 17 & 8.63 & 242 & 6.83 & 384 & 20.63 & 491 & 16.72 \\
\hline DENv234 & 17 & 7.61 & 287 & 5.72 & 384 & 20.12 & 571 & 16.24 \\
\hline DENv1234 & 28 & 11.94 & 212 & 10.05 & 343 & 21.27 & 411 & 18.47 \\
\hline
\end{tabular}

infections, leads to much lower optimal vaccination ages than if either ADE or cross-immunity play a role in disease severity. In this case all optimal vaccination ages apart from for only DENv2 existing are below 9 years of age, i.e. do not adhere to the licence restriction. If ADE or cross-immunity are considered many optimal vaccination ages are within the permitted age-range. However, some optimal ages in these cases are found to be above the limit of 45 years. This is even more pronounced if both ADE and cross-immunity play a role in disease severity.

Risk-free primary infections generally lead to higher optimal vaccination ages independent of whether secondary infections confer permanent cross-immunity. This is due to the fact that in this case vaccination does not necessarily need to take place before the primary infection occurs, in fact, since there is an increased risk in seronegative recipients it is best 
to vaccinate after a primary infection occurred. This is particularly obvious since in the case of only one serotype in existence vaccination is not recommended at all if primary infections are risk-free as there is no lifetime expected risk without vaccination. Vaccination is also not recommended for risk-free primary infections and asymptomatic third and fourth infections if DENv2 coexists with DENv3 or DENv4, and for risky primary infections and asymptomatic third and fourth infections for solely DENv1 or DENv2 existing. This is possibly due to the low vaccine efficacy against these serotypes compared to those for DENv3 and DENv4. Assuming asymptomatic third and fourth infections also results in higher optimal vaccination ages than the same scenarios would without cross-immunity. In this case the reason is that vaccinating seronegatives can have a negative effect, while vaccinating once most people have had an infection will mean that they are no longer at risk at all. Intuitively more serotypes coexisting leads to a higher lifetime expected risk. However, the optimal vaccination age tends to decrease when more serotypes circulate due to the higher combined force of infection.

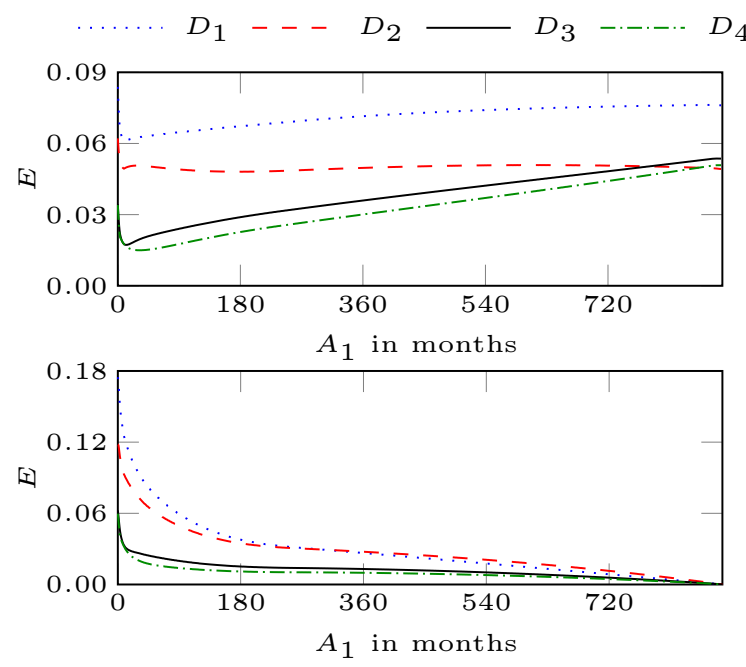

(a) The optimal vaccination age $A_{1}$ for DENv1,2,3 and 4 is 9 , 182,12 and 32 months for risky primary infections (top). For risk-free primary infections vaccination is not recommended (bottom).
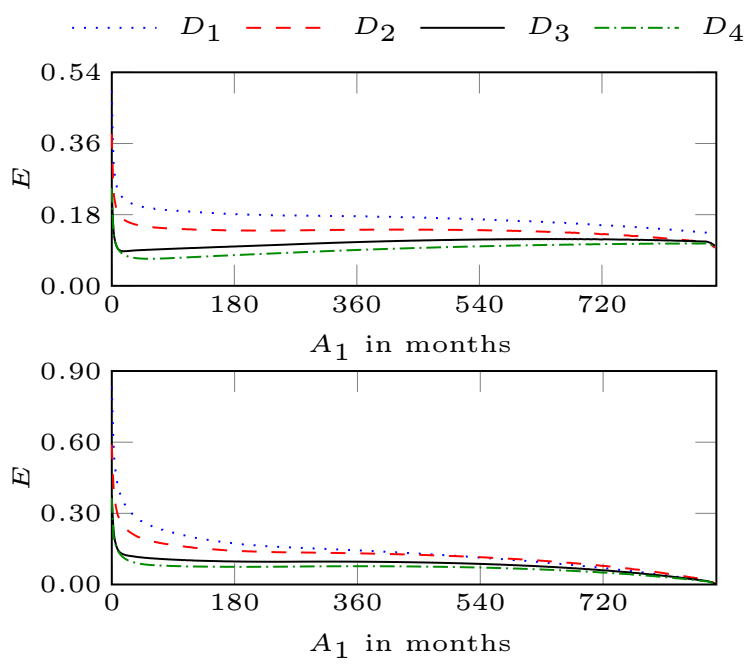

(b) The optimal vaccination age $A_{1}$ for DENv3 and 4 is 17 and 56 months for risky primary infections, while for DENv1 and 2 vaccination is not recommended (top). For risk-free primary infections vaccination is not recommended (bottom).

Figure 3: The lifetime expected risk $E$ in an endemic area with a single serotype as a function of age $A_{1}$ in months at which the first of three doses of vaccine is administered for constant efficacy as given in Table 3 where third and fourth infections are assumed (a) symptomatic, and (b) asymptomatic. The graphs at the top show results for risky primary infections, while those at the bottom show results for risk-free primary infections. The risk associated with an infection is serostatus-dependent and based on hospitalisation. 
We will now discuss the results for any combination of serotypes in more detail. The lifetime expected risk plotted against the age at which the first dose is given is shown in Figures 3 to 6 respectively for serotype combinations with one, two, three and four serotypes coexisting. In each of these figures the four graphs correspond to combinations of symptomatic post-secondary infections (subfigure (a)), asymptomatic post-secondary infections (subfigure (b)), risky primary infections (top graphs in subfigures (a) and (b)) and risk-free primary infections (bottom graphs in subfigures (a) and (b)). The optimal vaccination age is that at which the lifetime expected risk is minimal.

In an endemic area with only a single serotype in existence as shown in Figure 3 the optimal vaccination age, or whether the vaccine should be administered at all, highly depends on the assumptions relating to ADE and cross-immunity. Vaccination is not recommended if primary infections are risk-free since there is no lifetime expected risk without vaccination. Vaccination in this case has no effect in seropositive recipients and seronegative recipients if they are successfully vaccinated against the serotype in existence. It has a negative effect in seronegative recipients if they are not successfully vaccinated against that serotype but against other serotypes because the natural infection will now be a secondary type infection with an associated risk. This can be seen from the bottom graphs in the figure with the lifetime expected risk decreasing as the vaccination age increases. Clearly the likelihood of vaccinating seronegative recipients decreases as the age of vaccine recipients increases so that the overall risk is smaller for higher vaccination ages.

If primary infections are risky and third and fourth infections symptomatic the optimal vaccination ages vary between 9 and 182 months, with the highest optimal vaccination age for DENv2. On the other hand if third and fourth infections are asymptomatic vaccination in an endemic area with only DENv1 or DENv2 is not recommended and the optimal vaccination ages for DENv3 and DENv4 are respectively 17 and 56 months. In this case the efficacies play an important role in whether vaccination is recommended or not. The high efficacies against DENv3 and DENv4 mean that if the vaccine is administered early enough enough infections 
can be prevented and the risk is reduced. For DENv1 and DENv2 the vaccine efficacies are much lower and the increased risk in seronegative recipients leads to a negative effect if too few risky infections are prevented. Note that after a certain age the lifetime expected risk is a very flat function for asymptomatic third and fourth infections and risky primary infection for any serotype, i.e. the risk is influenced very little by the exact vaccination age after this point. The lifetime expected risk due to DENv1 is always highest due to the low vaccine efficacy against that serotype and the fact that the force of infection for DENv1 is highest. DENv2 leads to the second highest risk even though the force of infection for this serotype is lowest. In this case the low vaccine efficacy leads to few prevented cases and therefore a higher risk in comparison to DENv3 and DENv4. Only at very high vaccination ages is the risk due to DENv2 lower than that of DENv3 and DENv4 since at such high ages most infections will have already occurred and even with a high efficacy few cases can be prevented so that the force of infection becomes more relevant. DENv4 leads to the lowest risk for most vaccination ages due a relatively low force of infection and a high vaccine efficacy.

For two coexisting serotypes the results are presented in Figure 4 and it can be seen that vaccination is almost always recommended with the exception of risk-free primary infections and asymptomatic post-secondary infections for the combinations of DENv2 with DENv3 or DENv4. Again the reason for this is most likely the low vaccine efficacy and the increased risk in seronegative recipients if an infection occurs after vaccination. However, the lifetime expected risk in these cases is also a very flat function for older ages so that the lifetime expected risk is only slightly affected by vaccination in this age-range. The remaining vaccination ages are lowest for risky primary and symptomatic post-secondary infections, between 11 and 102 months, and highest for risk-free primary and asymptomatic post-secondary infections, between 618 and 696 months. It is noteworthy that in all considered scenarios serotype combinations including DENv1 have a higher lifetime expected risk than those without DENv1. This is particularly pronounced for risky primary infections. The reason for this is again the high force of infection but low vaccine efficacy for this serotype as was the case for an endemic 

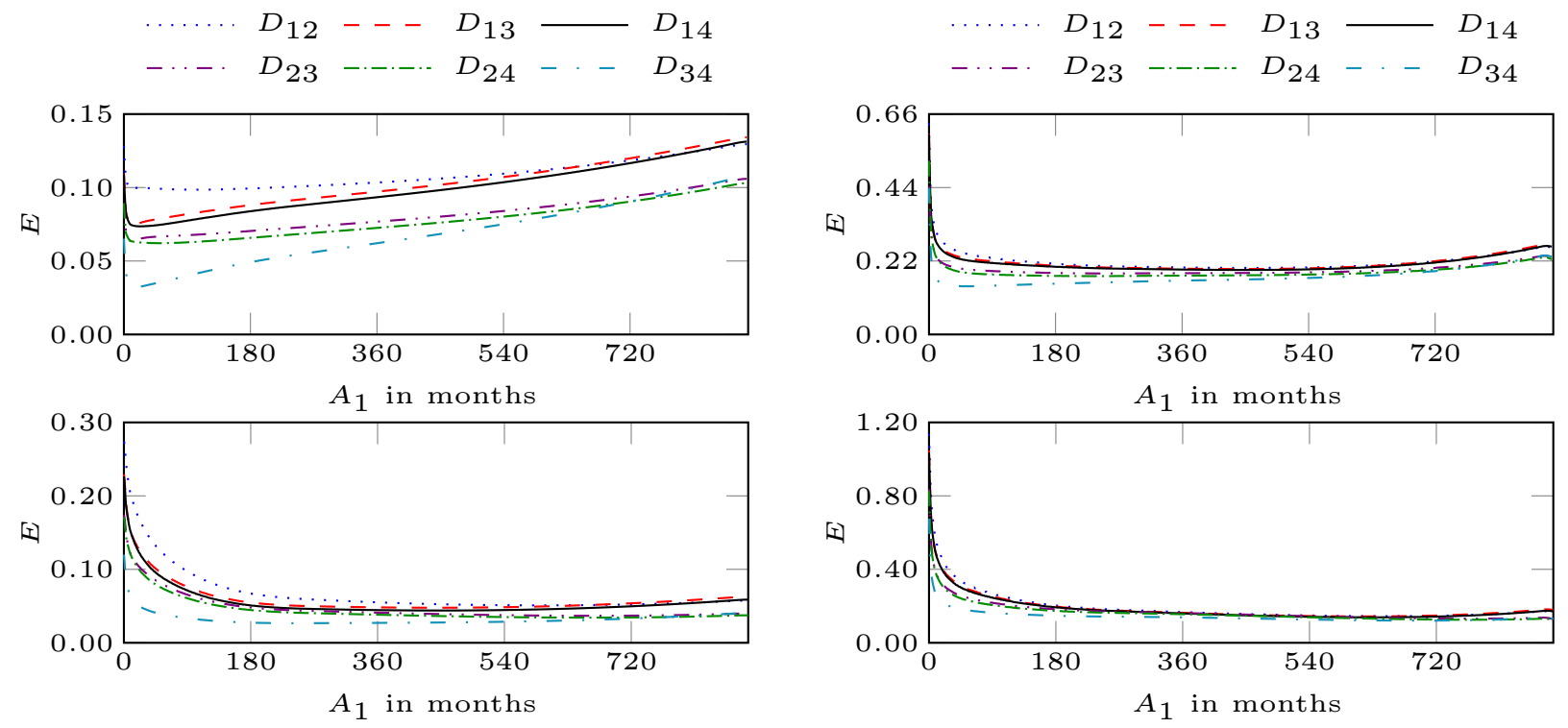

(a) The optimal vaccination age $A_{1}$ for DENv12, DENv13, DENv14, DENv23, DENv24, and DENv34 is 102, 11, 23, 11, 48 and 17 months for risky primary infections (top) amd 589, $445,449,667,640$, and 242 months for risk-free primary infections (bottom) respectively.

(b) The optimal vaccination age $A_{1}$ for DENv12, DENv13, DENv14, DENv23, DENv24, and DENv34 is 453, 445, 449, 253, 242 and 56 months for risky primary infections (top). For risk-free primary infections (bottom) the optimal vaccination age is $640,618,640$ and 696 months for DENv12, DENv13, DENv14 and DENv34 respectively while for DENv23 and DENv24 vaccination is not recommended.

Figure 4: The lifetime expected risk $E$ in an endemic area with two coexisting serotypes as a function of age $A_{1}$ in months at which the first of three doses of vaccine is administered for constant efficacy as given in Table 3 where third and fourth infections are assumed (a) symptomatic, and (b) asymptomatic. The graphs at the top show results for risky primary infections, while those at the bottom show results for risk-free primary infections. The risk associated with an infection is serostatus-dependent and based on hospitalisation.

area with only DENv1 circulating. Which combination causes the highest risk (DENv12) or the lowest risk (DENv34) for most vaccination ages can be explained similarly to the case of a single serotype in existence. Assuming all infections to be risky and symptomatic the lifetime expected risk increases significantly after the optimal vaccination age. There is a much wider range in which near optimal vaccination is possible otherwise, particularly if risk-free primary infections and asymptomatic post-secondary infections are considered. In this case only secondary infections are risky. Ideally vaccination takes place after most primary infections but few secondary infections have occurred so that there is no increased risk due to vaccinating seronegatives but many secondary infections can be prevented.

Figures 5 and 6 show the results for three and four coexisting serotypes. There are no longer any cases in which vaccination is not recommended. Similarly to two coexisting serotypes the optimal vaccination ages depend significantly on whether ADE or cross- 

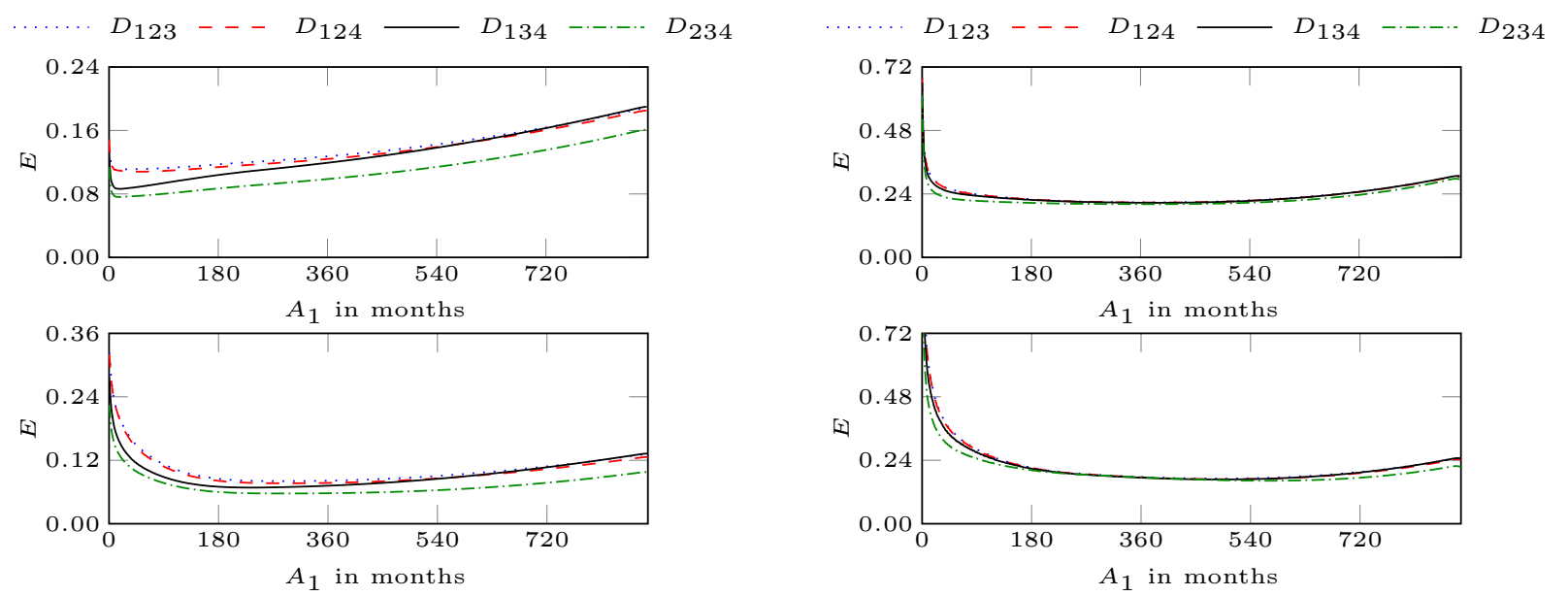

(a) The optimal vaccination age $A_{1}$ for DENv123, DENv124, DENv134, and DENv234 is 11, 48, 17 and 17 months for risky primary infections (top) and 287, 296, 242 and 287 months for risk-free primary infections (bottom) respectively.

(b) The optimal vaccination age $A_{1}$ for DENv123, DENv124, DENv134, and DENv234 is 384 months for risky primary infections (top). For risk-free primary infections (bottom) the optimal vaccination ages are 491, 499, 491 and 571 months respectively.

Figure 5: The lifetime expected risk $E$ in an endemic area with three coexisting serotypes as a function of age $A_{1}$ in months at which the first of three doses of vaccine is administered (a) for symptomatic and (b) for asymptomatic tertiary infections. The graphs at the top show results for risky primary infections, while those at the bottom show results for risk-free primary infections. The risk associated with an infection is serostatus-dependent and based on hospitalisation.

immunity are considered with the lowest ages (11-48 months) if neither is the case and the highest (411-571 months) if both are. For three coexisting serotypes serotype combinations including DENv1 result in a higher lifetime expected risk. Again this can be explained due to the high force of infection but low vaccine efficacy for this serotype. The observation for two coexisting serotypes regarding a wide range in which near optimal vaccination is possible particularly for risk-free primary and asymptomatic third and fourth infections is also true in the case of three and four serotypes.

\subsection{Licence Restrictions}

Many of the optimal vaccination ages are within the permitted age-range of 9-45 years for Dengvaxia. However, in the case of risky primary infections and symptomatic third and fourth infections all but one vaccination age is far below 9 years. For risky primary infections and asymptomatic third and fourth infections scenarios in which DENv3 or DENv4 exist alone or together also require vaccination below the age of 9 years to be ideal. On the other hand, for risk-free primary infections a number of serotype combinations lead to optimal vaccination 

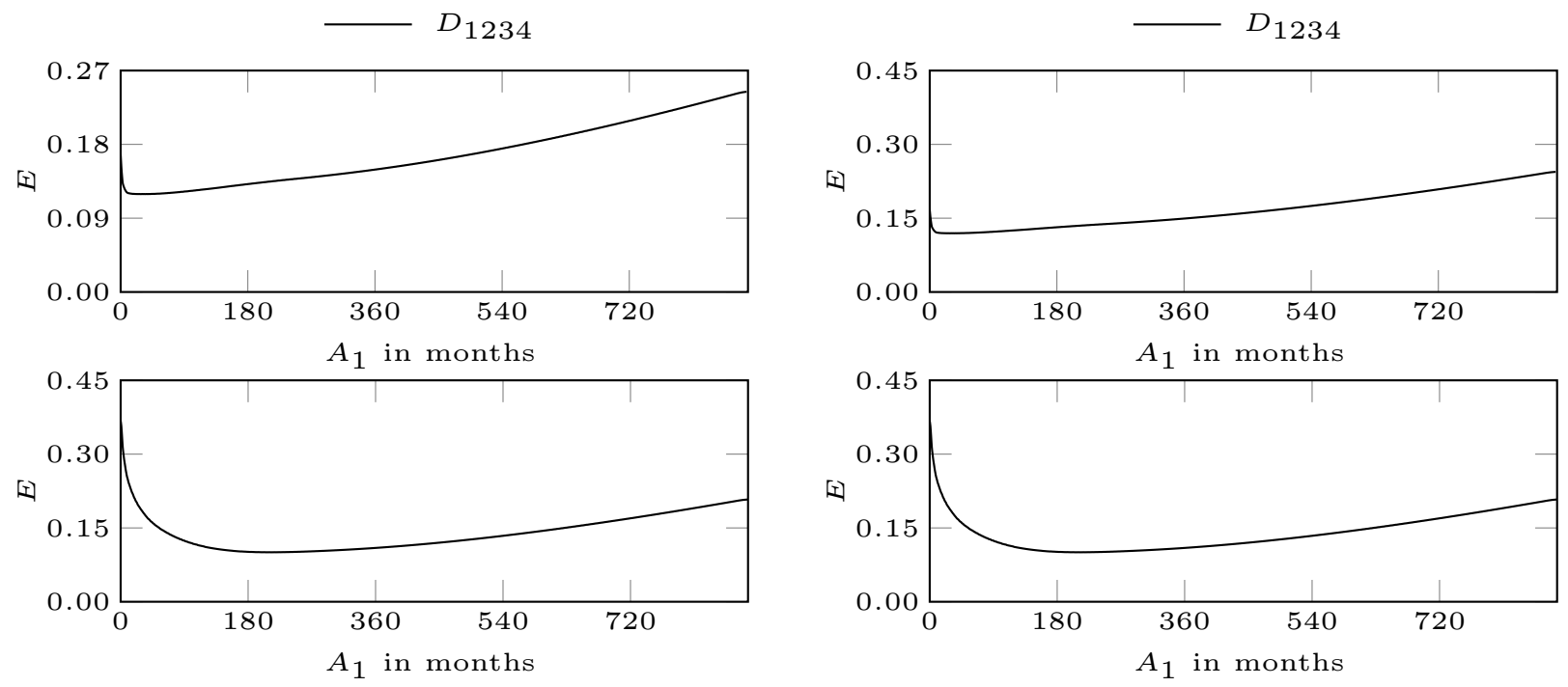

(a) The optimal vaccination age $A_{1}$ for DENv1234 is 28 months for risky primary infections (top) and 212 months for risk-free primary infections (bottom) respectively.

(b) The optimal vaccination age $A_{1}$ for DENv1234 is 343 months for risky primary infections (top) and 411 months for risk-free primary infections (bottom) respectively.

Figure 6: The lifetime expected risk $E$ in an endemic area with all serotypes co-circulating as a function of age $A_{1}$ in months at which the first of three doses of vaccine is administered (a) for symptomatic and (b) for asymptomatic tertiary and quaternary infections. The graphs at the top show results for risky primary infections, while those at the bottom show results for risk-free primary infections. The risk associated with an infection is serostatus-dependent and based on hospitalisation.

ages above the limit of 45 years. Additionally in some cases vaccination is not recommended at all, i.e. the vaccination age was determined to be above the maximum age for humans. If vaccination is restricted to 9-45 years for cases in which vaccination is recommended the vaccine should generally be administered as close to the optimal age as possible, i.e. at 9 years for optimal ages below the minimum age and at 45 years for optimal ages above the maximum age. This will lead to an increased lifetime expected risk in comparison to that achievable at the optimal age with the increase varying between less than $1 \%$ and $46 \%$. For most cases the increase is below $10 \%$ in comparison to the minimum. Vaccination is not recommended in the following situations:

(i) for any serotype existing by itself if primary infections are risk-free,

(ii) for either DENv1 or DENv2 existing separately if post-secondary infections are asymptomatic and primary infections risky, and

(iii) for the combinations of DENv2 and DENv3 or DENv2 and DENv4 when post-secondary 
infections are asymptomatic and primary infections risk-free.

If the vaccine is administered in theses cases it is best to vaccinate as late as possible, i.e. the final dose should be administered at 45 years. In situation (i) the minimal lifetime expected risk is zero. In situations (ii) and (iii) the lifetime expected risk is only slightly worsened by vaccination and the percentage increase in those cases varies between $13 \%$ and $45 \%$.

\section{Discussion}

We found the optimal vaccination age of a routine vaccination calendar for any combination of serotypes with the aim of minimising the risk of being hospitalised due to an infection with dengue. For the computations the serotype-specific forces of infection were derived by first determining the overall force of infection for dengue in Brazil according to serological data and then splitting it into the serotype-specific forces of infection according to the frequency of reported cases. The age-dependent pre-vaccine hospitalisation risk was estimated from data evaluated by Burattini et al. [23]. According to the reported increase in number of $\mathrm{SD}$ cases and hospitalisations in seronegative recipients in the long-term follow-up of Dengvaxia trials $[3,21,23]$ we considered the risk to be serostatus dependent based on vaccine and infection history. Additionally we investigated the effects of risk-free primary infections based on ADE and asymptomatic post-secondary infections based on cross-immunity after two heterologous infections. The vaccine efficacy was assumed constant and serotype-specific. For scenarios that resulted in an optimal vaccination age outwith the permitted age-range for Dengvaxia the achievable lifetime expected risk was compared to the minimum.

The optimal vaccination ages varied significantly with assumptions relating to ADE and cross-immunity as can be seen from Table 4. If neither ADE nor cross-immunity were considered the optimal vaccination ages varied between 9 and 182 months, almost all were below the permitted minimum age of 9 years. Vaccination was not recommended for risk-free primary infections and only a single serotype in circulation since in this case in the absence of vaccination the lifetime expected risk was zero. Vaccination in this case may lead to suc- 
cessful immunisation against a serotype that is not endemic prior to the individual acquiring an infection with the endemic serotype. This is harmful as there is an increased risk in initially seronegative recipients who are subsequently infected and who would not have had any risk without the vaccination. In some other scenarios vaccination was also not recommended due to the combination of low efficacies and increased risk in seronegative recipients in breakthrough cases. Assuming risk-free primary infections or asymptomatic third and fourth infections resulted in higher optimal vaccination ages, with the highest ages between 411 and 696 months for risk-free primary infections and asymptomatic post-secondary infections. The same assumptions also resulted in a wider range in which near optimal vaccination is possible for several coexisting serotypes. In general for risk-free primary infections vaccination should be administered after the first infection occurred to prevent an increased risk in seronegative recipients but ideally before most secondary infections so as to prevent as many infections as possible. This is particularly true if post-secondary infections are asymptomatic since vaccination after a secondary infection will no longer have any effect. The optimal vaccination age also depended on the number of serotypes with lower optimal ages as the number of serotypes increased. This is caused by the higher combined force of infection leading to a lower average age of infection.

Restricting the vaccination age to that permitted for Dengvaxia in Brazil resulted in a slight increase in lifetime expected risk in any case in which vaccination was recommended, usually below $10 \%$ from the optimum. When vaccination was ideally started below the minimum age of 9 years, under restriction it should be done as soon as possible after, i.e. at 9 years. On the other hand when vaccination was ideal above 45 years or was not recommended but carried out nonetheless it should be done as late as possible. Restricting the vaccination age mainly had negative effects if primary infections were risky and post-secondary infections symptomatic, in most other cases the vaccination ages were within the permitted age-range as long as vaccination was in fact recommended, or the increase effect was very small.

The results we obtained still leave many questions unanswered, particularly since the 
assumptions of ADE and cross-immunity significantly affect the optimal vaccination age. One of the most important questions is whether primary infections can indeed be considered risk-free. Based on nearly 7 million dengue cases in Brazil that caused the need for hospital treatment [23] our conclusion is that primary infections are probably risky. A second very important question is whether two heterologous infections confer permanent cross-immunity to all serotypes. This is usually assumed since third and fourth infections are rarely reported $[12,13]$. In this case the most relevant results would be those considering risky primary infections but asymptomatic third and fourth infections which have optimal vaccination ages almost exclusively within the permitted age-range of Dengvaxia. On the other hand if third and fourth infections are symptomatic and primary infections risky the optimal ages are below the minimum age for the vaccine. However, the minimum age of 9 years is based on the results from two large-scale clinical trials $[16,17]$ based on higher efficacy results above this age and a lower incidence of hospitalisation in comparison to younger recipients. It has since been disputed whether the higher efficacy is in fact caused by serostatus rather than age and long-term follow-up data has also shown that serostatus determines the higher hospitalisation incidence [20, 36-39]. The recommendations from the World Health Organisation and the labelling of Dengvaxia have therefore been adapted and it is now recommended to vaccinate individuals only above the age of 9 years after screening for a previous dengue infection $[3,21,22]$. However, it might be beneficial to also screen younger children and vaccinate those that are seropositive.

It should also be pointed out that the effect of lethality due to dengue was not considered in this paper. Clearly more accurate results could be obtained if the death rate were modelled realistically and the death due to dengue was included. The use of a step death function mortality rate has been used previously in work by internationally leading research groups to obtain results of practical significance in similar models [40-42]. However, on the one hand, the risk of lethality compared to that of hospitalisation is fairly small. Nunes et al. [43], for example, found a survival probability of 0.9995 in 30 years of dengue history in Brazil 
while Burattini et al. [23] evaluated the risk of hospitalisation over the period from 2000 to 2013 and found the risk of hospitalisation to average between 0.065 and 0.1047 . The risk of hospitalisation is therefore at least 120 times as high as that of lethality and should be the focus of research aiming at most effectively applying the new dengue vaccine. The conclusions regarding the differences between hospitalisation and lethality are supported by Maier [44] who fitted parametric functions to the age-dependent probability of requiring hospitalisation and the age-dependent risk of lethality for a dengue infection for the data from [23]. The risk of lethality does increase relative to the hospitalisation risk at very large ages (80-100 years) but it is still small in comparison to the risk of hospitalisation and this will have little impact on the results because few people will survive to this age and most of those that do will have experienced secondary dengue infection before that age. On the other hand, the focus on hospitalisation was chosen since hospitalisation is commonly used as the measure of dengue risk. Indeed hospitalisation is used as the measure mostly without reference to lethality. This is because the numbers are larger than for lethality and it is easier to evaluate dengue hospitalisation risk. For example the WHO SAGE Working Group on dengue vaccines uses hospitalisation risk to evaluate the risks of dengue [3], as do Aguiar and Stollenwerk [37] and Aguiar et al. [20], Ferguson et al. [45], Martínez-Vega et al. [46], Wilder-Smith et al. [47] and Halstead and Russell [18]. The dengue vaccine WHO position paper [21] also uses hospitalisation risk to estimate vaccine risk. In future work it may however be beneficial to take a closer look at the risk of lethality as well.

\section{Acknowledgements}

DG and SM are grateful to the University of Strathclyde for support for a PhD studentship. DG is grateful to the Leverhulme Trust for support from a Leverhulme Fellowship (RF-2015-88) and the British Council Malaysia for funding from the Dengue Tech Challenge (Application Reference DTC 16022). EM and DG are grateful to the Science Without Borders Program for a Special Visiting Fellowship (CNPq grant 30098/2014/7). MNB is grateful 
to the Brazilian Ministry of Health for grant 777588/2012.

\section{Appendix A. Estimation of $\xi_{1}: \xi_{2}: \xi_{3}: \xi_{4}$}

In order to obtain serotype-specific forces of infection from Equation (11) we need to estimate the ratios $\xi_{1}: \xi_{2}: \xi_{3}: \xi_{4}$. Note that the larger $\xi_{i}$ the larger $\lambda_{0}^{i}(a)$ and thus the larger the number of cases of serotype $i$. For the first infection an unaffected individual will catch dengue serotype $i$ according to a time which has an exponential distribution with rate $\lambda_{0}^{i}(a) C_{i}(a)$. Hence, if all four serotypes are present, the probability that the individual is affected by serotype $i$ is

$$
\frac{\lambda_{0}^{i}(a) C_{i}(a)}{\lambda_{0}^{1}(a) C_{1}(a)+\lambda_{0}^{2}(a) C_{2}(a)+\lambda_{0}^{3}(a) C_{3}(a)+\lambda_{0}^{4}(a) C_{4}(a)} .
$$

The duration of protection by dengue antibodies is quite short, and there is not much variation between the average duration of protection by maternal antibodies for different serotypes [29] so as an approximation we may assume that $C_{i}(a)=C(a)$ is independent of serotype. Using Equation (16) we deduce that the probability that this individual is recorded as a case of serotype $i$ is $\xi_{i} /\left(\xi_{1}+\xi_{2}+\xi_{3}+\xi_{4}\right)$. Hence in this situation it is reasonable to make the assumption that the ratio $\xi_{1}: \xi_{2}: \xi_{3}: \xi_{4}$ is the same as $n_{1}: n_{2}: n_{3}: n_{4}$ the ratio of the number of cases of each serotype.

This is just an approximation as the situation is more complex for second and subsequent infections. However serotypes more recently introduced into Brazil will be under-represented at the start of the data because they were not present then, but over-represented at the end of the data because some individuals will have immunity to the other serotypes which have been present for longer. Also $q(a)$, hence $\lambda_{0}^{i}(a)(i=1,2,3,4)$, is higher at younger ages where secondary infections are not as important. Additionally, if we make the plausible assumption that secondary cases (apart from ADE) are less likely to be symptomatic because the first infection builds up partial immunity and that the recorded cases will thus mainly correspond to primary infections, the above approximation will be valid. As there is no other obvious 
simple way to estimate the parameters $\xi_{1}, \xi_{2}, \xi_{3}$ and $\xi_{4}$ from the observed data contained in the number of dengue cases we use the above method as a simple approximation.

\section{Appendix B. Serostatus-dependent Risk Functions}

In order to incorporate the serostatus-dependent risk according to the data presented in Table 2 in the risk function for any infection and vaccination history we need to consider four different cases based on whether ADE leads to risk-free primary infections and whether two heterologous infections confer permanent cross-immunity.

In the case of risky primary infections we denote the risk of hospitalisation for at risk unvaccinated seronegatives, unvaccinated seropositives and successfully vaccinated initially seronegatives of age $a$ relative to the risk of hospitalisation for at risk unvaccinated seronegatives aged $a$ by $\bar{h}^{-}(a), \bar{h}^{+}(a)$ and $h_{*}^{-}(a)$ respectively. Further the risk for at risk successfully vaccinated initially seropositives relative to at risk unvaccinated seropositives is denoted by $h_{*}^{+}(a)$. For risk-free primary infections we instead define the corresponding risks relative to at risk unvaccinated seropositive individuals aged $a$ and denote them by $\bar{g}^{-}(a), \bar{g}^{+}(a), g_{*}^{-}(a)$ and $g_{*}^{+}(a)$ respectively. Note that $g_{*}^{+}(a)=h_{*}^{+}(a)$. We consider only at risk individuals, e.g. in the case of risky primary infections and no cross-immunity only individuals with antibodies (due to infection and vaccination) to no more than three serotypes are at risk, whereas in the case of risk-free primary infections and permanent cross-immunity only individuals with antibodies to exactly one serotype are at risk. Further the relative risks $h_{*}^{-}(a), g_{*}^{-}(a), h_{*}^{+}(a)$ and $g_{*}^{+}(a)$ are for successfully vaccinated individuals only, while unsuccessfully vaccinated individuals are assumed to have the same risk as unvaccinated individuals with the same serostatus. The data presented in Table 2 therefore needs to be adjusted for individuals being at risk and successfully vaccinated. Note that the data considers only individuals aged 2-16 years. This age-class will be denoted by $G_{0}$. The above relative risks can be determined only for $a \in G_{0}$ which, due to the nature of the data, is constant across the entire age-class.

We define $p_{1}\left(G_{0}\right)$ as the probability that an unvaccinated initial seropositive of age $a \in G_{0}$ 
is at risk at the pre-vaccine steady-state, $p_{2}$ as the probability for an initially seronegative individual to be successfully vaccinated but remain at risk immediately after vaccination and $p_{3}\left(G_{0}\right)$ as the probability for an initially seropositive individual of age $a \in G_{0}$ to be successfully vaccinated but remain at risk immediately after vaccination. Note that $p_{1}\left(G_{0}\right), p_{2}$ and $p_{3}\left(G_{0}\right)$ depend on whether two heterologous infections confer permanent cross-immunity. In the case of symptomatic third and fourth infections $p_{1}\left(G_{0}\right)$ is simply the probability that a seropositive individual is seropositive to at most three serotypes. The steady-state probability of being seropositive to serotype $i$ at age $a$ before a vaccine is introduced, i.e. $u_{i}^{0}(a)$, yields the proportion of individuals in the age-class $G_{0}$ who are unaffected by serotype $i$ as

$$
u_{i}^{0}\left(G_{s}\right)=\frac{\frac{N_{H}}{L} \int_{2}^{16} u_{i}^{0}(a) \pi_{H}(a) d a}{\frac{N_{H}}{L} \int_{2}^{16} \pi_{H}(a) d a},
$$

where the integration limits are obtained from the limits of age-class $G_{0}$. Therefore

$$
p_{1}\left(G_{0}\right)=\frac{1-u_{1}^{0}\left(G_{0}\right) u_{2}^{0}\left(G_{0}\right) u_{3}^{0}\left(G_{0}\right) u_{4}^{0}\left(G_{0}\right)-\left(1-u_{1}^{0}\left(G_{0}\right)\right)\left(1-u_{2}^{0}\left(G_{0}\right)\right)\left(1-u_{3}^{0}\left(G_{0}\right)\right)\left(1-u_{4}^{0}\left(G_{0}\right)\right)}{1-u_{1}^{0}\left(G_{0}\right) u_{2}^{0}\left(G_{0}\right) u_{3}^{0}\left(G_{0}\right) u_{4}^{0}\left(G_{0}\right)} .
$$

An at risk successfully vaccinated initially seronegative individual must have been vaccinated against one, two or three serotypes so that with $v_{e}^{i}$ denoting the vaccine efficacy against serotype $i$ and $v_{e}^{-}$the overall vaccine efficacy for seronegative recipients we obtain

$$
p_{2}=v_{e}^{-} \frac{1-v_{e}^{1} v_{e}^{2} v_{e}^{3} v_{e}^{4}-\left(1-v_{e}^{1}\right)\left(1-v_{e}^{2}\right)\left(1-v_{e}^{3}\right)\left(1-v_{e}^{4}\right)}{1-\left(1-v_{e}^{1}\right)\left(1-v_{e}^{2}\right)\left(1-v_{e}^{3}\right)\left(1-v_{e}^{4}\right)} .
$$

For cross-immunity only individuals seropositive to at most one serotype are at risk, thus

and

$$
\begin{aligned}
p_{1}\left(G_{0}\right) & =\frac{\sum_{i=1}^{4}\left[\left(1-u_{i}^{0}\left(G_{0}\right)\right) u_{j}^{0}\left(G_{0}\right) u_{k}^{0}\left(G_{0}\right) u_{l}^{0}\left(G_{0}\right)\right]}{1-u_{1}^{0}\left(G_{0}\right) u_{2}^{0}\left(G_{0}\right) u_{3}^{0}\left(G_{0}\right) u_{4}^{0}\left(G_{0}\right)}, \\
p_{2} & =v_{e}^{-} \frac{\sum_{i=1}^{4}\left[v_{e}^{i}\left(1-v_{e}^{j}\right)\left(1-v_{e}^{k}\right)\left(1-v_{e}^{l}\right)\right]}{1-\left(1-v_{e}^{1}\right)\left(1-v_{e}^{2}\right)\left(1-v_{e}^{3}\right)\left(1-v_{e}^{4}\right)} .
\end{aligned}
$$

To compute $p_{3}\left(G_{0}\right)$ in the case of no permanent cross-immunity we need to consider the distribution of seropositivity to exactly one or two serotypes in age-class $G_{0}$, i.e. 
and

$$
\begin{aligned}
s_{i}\left(G_{0}\right) & =\frac{\left(1-u_{i}^{0}\left(G_{0}\right)\right) u_{j}^{0}\left(G_{0}\right) u_{k}^{0}\left(G_{0}\right) u_{l}^{0}\left(G_{0}\right)}{1-u_{i}^{0}\left(G_{0}\right) u_{j}^{0}\left(G_{0}\right) u_{k}^{0}\left(G_{0}\right) u_{l}^{0}\left(G_{0}\right)}, \\
s_{i j}\left(G_{0}\right) & =\frac{\left(1-u_{i}^{0}\left(G_{0}\right)\right)\left(1-u_{j}^{0}\left(G_{0}\right)\right) u_{k}^{0}\left(G_{0}\right) u_{l}^{0}\left(G_{0}\right)}{1-u_{i}^{0}\left(G_{0}\right) u_{j}^{0}\left(G_{0}\right) u_{k}^{0}\left(G_{0}\right) u_{l}^{0}\left(G_{0}\right)} .
\end{aligned}
$$

Then the probabilities of being initially seropositive to exactly one serotype and subsequently vaccinated against at most two other serotypes, or initially seropositive to exactly two serotypes and subsequently being vaccinated against at most one other serotype are respectively

and

$$
\begin{aligned}
& p_{r}^{i}\left(G_{0}\right)=s_{i}\left(G_{0}\right) v_{e}^{+} \frac{1-v_{e}^{j} v_{e}^{k} v_{e}^{l}-\left(1-v_{e}^{j}\right)\left(1-v_{e}^{k}\right)\left(1-v_{e}^{l}\right)}{1-\left(1-v_{e}^{j}\right)\left(1-v_{e}^{k}\right)\left(1-v_{e}^{l}\right)}, \\
& p_{r}^{i j}\left(G_{0}\right)=s_{i j}\left(G_{0}\right) v_{e}^{+} \frac{1-v_{e}^{k} v_{e}^{l}-\left(1-v_{e}^{k}\right)\left(1-v_{e}^{l}\right)}{1-\left(1-v_{e}^{k}\right)\left(1-v_{e}^{l}\right)} .
\end{aligned}
$$

Here $v_{e}^{+}$denotes the overall vaccine efficacy for seropositive individuals. Hence the probability of being initially seropositive and then successfully vaccinated but remaining at risk is given by

$$
p_{3}\left(G_{0}\right)=\sum_{i=1}^{4} p_{r}^{i}\left(G_{0}\right)+\sum_{\substack{i, j=1 \\ i \neq j}}^{4} p_{r}^{i j}\left(G_{0}\right) .
$$

Using these probabilities and Table 2 we know that of the 988 seropositives in the control group only $988 p_{1}\left(G_{0}\right)$ were at risk and of the 2,027 seropositives in the vaccine group only $2,027 p_{3}\left(G_{0}\right)$ were at risk. Further of the 712 seronegatives in the vaccine group $712\left(1-v_{e}^{-}\right)$ were not successfully vaccinated and therefore $712\left(1-v_{e}^{-}\right) \frac{9}{377}$ of the 24 recorded hospitalisation cases in the vaccine group occurred in individuals for whom the vaccine was not effective. Thus for $a \in G_{0}$

$$
\begin{aligned}
\bar{h}^{-}(a) & =1, & \bar{g}^{-}(a) & =0, \\
\bar{h}^{+}(a) & =\frac{26}{988 p_{1}\left(G_{0}\right)} / \frac{9}{377}, & \bar{g}^{+}(a) & =1, \\
h_{*}^{-}(a) & =\frac{24-712\left(1-v_{e}^{-}\right) \frac{9}{377}}{712 p_{2}} / \frac{9}{377}, & g_{*}^{-}(a) & =\frac{24-712\left(1-v_{e}^{-}\right) \frac{9}{377}}{712 p_{2}} / \frac{26}{988 p_{1}\left(G_{0}\right)},
\end{aligned}
$$


and

$$
h_{*}^{+}(a)=g_{*}^{+}(a)= \begin{cases}0, & \text { for permanent cross-immunity } \\ \frac{16}{2,027 p_{3}\left(G_{0}\right)} / \frac{26}{988 p_{1}\left(G_{0}\right)}, & \text { otherwise. }\end{cases}
$$

These relative risks together with the pre-vaccine risk function $R(a)$ given in Equation (24) can now be used to determine the risk functions associated with any combination of prior infections and successful vaccination. However, it needs to be noted that the relative risks are determined for $a \in G_{0}$ only. We will therefore assume that the relative risk for individuals aged $a \notin G_{0}$ is the same as that for those aged $a \in G_{0}$. In the case of individuals who have experienced a natural infection with one or more serotypes at some point and have been successfully vaccinated against at least one other serotype we do not consider the order of infection or vaccination to be relevant and assume the risk to be that of an initial seropositive who was successfully vaccinated. We let $R^{-}(a)$ be the pre-vaccine risk for seronegatives and $R^{+}(a)$ the pre-vaccine risk for seropositives. Then for risky primary infections and no crossimmunity

$$
\begin{aligned}
R(a)=\left\{u_{1}^{0}(a)\right. & u_{2}^{0}(a) u_{3}^{0}(a) u_{4}^{0}(a)+\left[1-u_{1}^{0}(a) u_{2}^{0}(a) u_{3}^{0}(a) u_{4}^{0}(a)\right. \\
& \left.\left.-\left(1-u_{1}^{0}(a)\right)\left(1-u_{2}^{0}(a)\right)\left(1-u_{3}^{0}(a)\right)\left(1-u_{4}^{0}(a)\right)\right] \bar{h}^{+}(a)\right\} R^{-}(a),
\end{aligned}
$$

and

$$
\begin{aligned}
& R_{i \bar{j} \bar{k} \bar{l}}(a)=\bar{h}^{-}(a) R^{-}(a)=R^{-}(a), \\
& R_{i j k l}(a)=R_{i j k \bar{l}}(a)=R_{i j \bar{k} \bar{l}}(a)=\bar{h}^{+}(a) R^{-}(a), \\
& R_{i j_{*} k_{*} l_{*}}(a)=R_{i j_{*} k_{*} \bar{l}}(a)=R_{i j_{*} \bar{k} \bar{l}}(a)=h_{*}^{-}(a) R^{-}(a), \\
& R_{i j k l_{*}}(a)=R_{i j k_{*} l_{*}}(a)=R_{i j k_{*} \bar{l}}(a)=h_{*}^{+}(a) R^{+}(a)=h_{*}^{+}(a) \bar{h}^{+}(a) R^{-}(a) .
\end{aligned}
$$


For risky primary infection and cross-immunity

$$
R(a)=\left\{u_{1}^{0}(a) u_{2}^{0}(a) u_{3}^{0}(a) u_{4}^{0}(a)+\sum_{i=1}^{4}\left[\left(1-u_{i}^{0}(a)\right) u_{j}^{0}(a) u_{k}^{0}(a) u_{l}^{0}(a)\right] \bar{h}^{+}(a)\right\} R^{-}(a),
$$

and

$$
\begin{aligned}
& R_{i \bar{j} \bar{k} \bar{l}}(a)=\bar{h}^{-}(a) R^{-}(a)=R^{-}(a), \\
& R_{i j \bar{k} \bar{l}}(a)=\bar{h}^{+}(a) R^{-}(a), \\
& R_{i j_{*} \bar{k} \bar{l}}(a)=h_{*}^{-}(a) R^{-}(a), \\
& R_{i j k l}(a)=R_{i j k \bar{l}}(a)=R_{i j_{*} k_{*} l_{*}}(a)=R_{i j_{*} k_{*} \bar{l}}(a)=R_{i j k l_{*}}(a)=R_{i j k_{*} l_{*}}(a)=R_{i j k_{*} \bar{l}}(a)=0 .
\end{aligned}
$$

In the case of risk-free primary infections $R^{-}(a)=0$ so if there is no cross-immunity

$$
R(a)=\left[1-u_{1}^{0}(a) u_{2}^{0}(a) u_{3}^{0}(a) u_{4}^{0}(a)-\left(1-u_{1}^{0}(a)\right)\left(1-u_{2}^{0}(a)\right)\left(1-u_{3}^{0}(a)\right)\left(1-u_{4}^{0}(a)\right)\right] R^{+}(a),
$$

and

$$
\begin{aligned}
& R_{i \bar{j} \bar{k} \bar{l}}(a)=0, \\
& R_{i j k l}(a)=R_{i j k \bar{l}}(a)=R_{i j \bar{k} \bar{l}}(a)=\bar{g}^{+}(a) R^{+}(a)=R^{+}(a), \\
& R_{i j_{*} k_{*} l_{*}}(a)=R_{i j_{*} k_{*} \bar{l}}(a)=R_{i j_{*} \bar{k} \bar{l}}(a)=g_{*}^{-}(a) R^{+}(a), \\
& R_{i j k l_{*}}(a)=R_{i j k_{*} l_{*}}(a)=R_{i j k_{*} \bar{l}}(a)=g_{*}^{+}(a) R^{+}(a) .
\end{aligned}
$$

And lastly for risk-free primary infections and cross-immunity

$$
R(a)=\sum_{i=1}^{4}\left[\left(1-u_{i}^{0}(a)\right) u_{j}^{0}(a) u_{k}^{0}(a) u_{l}^{0}(a)\right] R^{+}(a),
$$

and

$$
\begin{aligned}
& R_{i j \bar{k} \bar{l}}(a)=\bar{g}^{+}(a) R^{+}(a)=R^{+}(a), \\
& R_{i j_{*} \bar{k} \bar{l}}(a)=g_{*}^{-}(a) R^{+}(a), \\
& R_{i j k l}(a)=R_{i j k \bar{l}}(a)=R_{i \bar{j} \bar{k} \bar{l}}(a)=0, \\
& R_{i j_{*} k_{*} l_{*}}(a)=R_{i j_{*} k_{*} \bar{l}}(a)=R_{i j k l_{*}}(a)=R_{i j k_{*} l_{*}}(a)=R_{i j k_{*} \bar{l}}(a)=0 .
\end{aligned}
$$

\section{References}

[1] World Health Organization, Global strategy for dengue prevention and control 2012-2020, World Health Organization, Geneva, Switzerland, 2012.

[2] S. Bhatt, P. W. Gething, O. J. Brady, J. P. Messina, A. W. Farlow, C. L. Moyes, J. M. Drake, J. S. Brownstein, A. G. Hoen, O. Sankoh, M. F. Myers, D. B. George, T. Jaenisch, G. R. W. Wint, C. P. Simmons, T. W. Scott, J. J. Farrar, S. I. Hay, The global distribution and burden of dengue, Nature 496 (2013) 504-507.

[3] SAGE Working Group on Dengue Vaccines and WHO Secretariat, Background paper on dengue vaccines, World Health Organization, Geneva, Switzerland, 2016. 
[4] V. E. M. de Araújo, J. M. T. Bezerra, F. F. Amâncio, V. M. de Azeredo Passos, M. Carneiro, Increase in the burden of dengue in Brazil and federated units, 2000 and 2015: analysis of the global burden of disease study 2015, Revista Brasileira de Epidemiologia 20 (2017) 205-216.

[5] M. T. de Oliveira Mota, A. C. Terzian, M. L. C. R. Silva, C. Estofolete, M. L. Nogueira, Mosquito-transmitted viruses - the great Brazilian challenge, Brazilian Journal of Microbiology 47 (2016) 38-50.

[6] P. Kotsakiozi, A. Gloria-Soria, A. Caccone, B. Evans, R. Schama, A. J. Martins, J. R. Powell, Tracking the return of Aedes aegypti to Brazil, the major vector of the dengue, chikungunya and zika viruses, PLoS Neglected Tropical Diseases 11 (2017) e0005653.

[7] R. C. Fares, K. P. Souza, G. Añez, M. Rios, Epidemiological scenario of dengue in Brazil, BioMed Research International 2015 (2015).

[8] S. B. Halstead, Dengue haemorrhagic fever - a public health problem and a field for research, Bulletin of the World Health Organization 58 (1980) 1-21.

[9] S. B. Halstead, Antibodies determine virulence in dengue, Annals of the New York Academy of Sciences 1171 (2009) E48-E56.

[10] A. S.-Y. Leong, K. T. Wong, T. Y.-M. Leong, P. H. Tan, P. Wannakrairot, The pathology of dengue hemorrhagic fever, Seminars in Diagnostic Pathology 24 (2007) 227-236.

[11] A. Jain, U. C. Chaturvedi, Dengue in infants: an overview, FEMS Immunology \& Medical Microbiology 59 (2010) 119-130.

[12] J. R. Fried, R. V. Gibbons, S. Kalayanarooj, S. J. Thomas, A. Srikiatkhachorn, I.-K. Yoon, R. G. Jarman, S. Green, A. L. Rothman, D. A. Cummings, Serotype-specific differences in the risk of dengue hemorrhagic fever: an analysis of data collected in Bangkok, Thailand from 1994 to 2006, PLoS Neglected Tropical Diseases 4 (2010) e617.

[13] R. V. Gibbons, S. Kalanarooj, R. G. Jarman, A. Nisalak, D. W. Vaughn, T. P. Endy, M. P. Mammen, A. Srikiatkhachorn, Analysis of repeat hospital admissions for dengue to estimate the frequency of third or fourth dengue infections resulting in admissions and dengue hemorrhagic fever, and serotype sequences, The American Journal of Tropical Medicine and Hygiene 77 (2007) 910-913.

[14] Sanofi Pasteur Press Release, Dengvaxia, World's first dengue vaccine, approved in Mexico, 2015. https://www.sanofipasteur.com/en/media-room/press-releases/ (last accessed: May 23 2018).

[15] Sanofi Pasteur Press Release, First dengue vaccine approved in more than 10 countries, 2016. https://www.sanofipasteur.com/en/media-room/press-releases/ (last accessed: May 23 2018).

[16] M. R. Capeding, N. H. Tran, S. R. S. Hadinegoro, H. I. H. J. M. Ismail, T. Chotpitayasunondh, M. N. Chua, C. Q. Luong, K. Rusmil, D. N. Wirawan, R. Nallusamy, P. Pitisuttithum, U. Thisyakorn, I. K. Yoon, D. van der Vliet, E. Langevin, T. Laot, Y. Hutagalung, C. Frago, M. Boaz, T. A. Wartel, N. G. Tornieporth, M. Saville, A. Bouckenooghe, the CYD14 
Study Group, Clinical efficacy and safety of a novel tetravalent dengue vaccine in healthy children in Asia: a phase 3, randomised, observer-masked, placebo-controlled trial, The Lancet 384 (2014) 1358-1365.

[17] S. R. Hadinegoro, J. L. Arredondo-García, M. R. Capeding, C. Deseda, T. Chotpitayasunondh, R. Dietze, H. I. H. J. M. Ismail, H. Reynales, K. Limkittikul, D. M. Rivera-Medina, H. N. Tran, A. Bouckenooghe, D. Chansinghakul, M. Corts, K. Fanouillere, R. Forrat, C. Frago, S. Gailhardou, N. Jackson, F. Noriega, E. Plennevaux, T. A. Wartel, B. Zambrano, M. Saville, for the CYD-TDV Dengue Vaccine Working Group, Efficacy and long-term safety of a dengue vaccine in regions of endemic disease, New England Journal of Medicine 373 (2015) 1195-1206.

[18] S. B. Halstead, P. K. Russell, Protective and immunological behavior of chimeric yellow fever dengue vaccine, Vaccine 34 (2016) 1643-1647.

[19] M. Aguiar, S. B. Halstead, N. Stollenwerk, The impact of the newly licensed dengue vaccine in endemic countries, PLoS Neglected Tropical Diseases 10 (2016) e0005179.

[20] M. Aguiar, N. Stollenwerk, S. B. Halstead, The risks behind Dengvaxia recommendation, The Lancet Infectious Diseases 16 (2016) 882-883.

[21] SAGE Working Group on Dengue Vaccines and WHO Secretariat, Background paper on dengue vaccines - revision of the background paper from 17 March 2016, 2018.

[22] Sanofi Pasteur Communication, Sanofi Pasteur communication on SAGE recommendation about Dengvaxia dengue vaccine, 2018. http://mediaroom.sanofi.com/sanofi-pasteurcommunication-on-sage-recommendation-about-dengvaxia-dengue-vaccine/ (last accessed: May 23 2018).

[23] M. N. Burattini, L. F. Lopez, F. A. B. Coutinho, J. B. Siqueira Jr, S. Homsani, E. Sarti, E. Massad, Age and regional differences in clinical presentation and risk of hospitalization for dengue in Brazil, 2000-2014, Clinics 71 (2016) 455-463.

[24] H. W. Hethcote, Optimal ages of vaccination for measles, Mathematical Biosciences 89 (1988) $29-52$.

[25] S. B. Maier, X. Huang, E. Massad, M. Amaku, M. N. Burattini, D. Greenhalgh, Analysis of the optimal vaccination age for dengue in Brazil with a tetravalent dengue vaccine, Mathematical Biosciences 294 (2017) 15-32.

[26] United States Central Intelligence Agency, Brazil - The World Factbook, 2016. https: //www.cia.gov/library/publications/the-world-factbook/geos/br.html (last accessed: November 21 2016).

[27] E. Massad, F. Coutinho, M. Burattini, M. Amaku, Estimation of $R_{0}$ from the initial phase of an outbreak of a vector-borne infection, Tropical Medicine and International Health 15 (2010) $120-126$.

[28] C. Favier, N. Dégallier, M. G. Rosa-Freitas, J.-P. Boulanger, J. R. Costa Lima, J. F. LuitgardsMoura, C. E. Menkès, B. Mondet, C. Oliveira, E. T. S. Weimann, P. Tsouris, Early determination of the reproductive number for vector-borne diseases: the case of dengue in Brazil, Tropical Medicine and International Health 11 (2006) 332-340. 
[29] W. G. van Panhuis, C. Luxemburger, K. Pengsaa, K. Limikittikul, A. Sabchareon, J. Lang, A. P. Durbin, D. A. T. Cummings, Decay and persistence of maternal dengue antibodies among infants in Bangkok, American Society of Tropical Medicine and Hygiene 85 (2011) 355-362.

[30] C. Farrington, Modelling forces of infection for measles, mumps and rubella, Statistics in Medicine 9 (1990) 953-967.

[31] M. N. Burattini, Unpublished serological data, Personal Communication, 2017.

[32] T. N. B. Chau, N. T. Hieu, K. L. Anders, M. Wolbers, L. B. Lien, L. T. M. Hieu, T. T. Hien, N. T. Hung, J. Farrar, S. Whitehead, C. P. Simmons, Dengue virus infections and maternal antibody decay in a prospective birth cohort study of Vietnamese infants, The Journal of Infectious Diseases 200 (2009) 1893-1900.

[33] M. Amaku, L. Coudeville, E. Massad, Designing a vaccination strategy against dengue, Revista do Instituto de Medicina Tropical de Sao Paulo 54 (2012) S18-S21.

[34] M. N. Burattini, Unpublished serological data. Taken from PNCD/SVS/MS Brazil., 2019. https://portalms.saude.gov.br/saude-de-a-z/dengue/situacao-epidemiologicadados (last accessed: August 2 2018).

[35] R. Ximenes, M. Amaku, L. F. Lopez, F. A. B. Coutinho, M. N. Burattini, D. Greenhalgh, A. Wilder-Smith, C. J. Struchiner, E. Massad, The risk of dengue for non-immune foreign visitors to the 2016 summer Olympic Games in Rio de Janeiro, Brazil, BMC Infectious Diseases 16 (2016) Article No. 186.

[36] M. Aguiar, N. Stollenwerk, Dengvaxia: age as surrogate for serostatus, The Lancet Infectious Diseases 18 (2018) 245.

[37] M. Aguiar, N. Stollenwerk, Dengvaxia efficacy dependency on serostatus: a closer look at more recent data, Clinical Infectious Diseases 66 (2018) 641-642.

[38] A. L. Dans, L. F. Dans, M. A. D. Lansang, M. A. A. Silvestre, G. H. Guyatt, Controversy and debate on dengue vaccine series-paper 1: review of a licensed dengue vaccine: inappropriate subgroup analyses and selective reporting may cause harm in mass vaccination programs, Journal of Clinical Epidemiology 95 (2018) 137.

[39] S. B. Halstead, Critique of World Health Organization recommendation of a dengue vaccine, Journal of Infectious Diseases: Perspectives 241 (2016) 1793-1795.

[40] R. M. Anderson, R. M. May, Spatial, temporal and genetic heterogeneity in host populations and the design of immunisation programmes, IMA Journal of Mathematics Applied in Biology and Medicine 1 (1984) 233-266.

[41] R. M. Anderson, R. M. May, Age-related changes in the rate of disease transmission: implications for the design of vaccination programmes, Cambridge Journal of Hygiene 94 (1985) $365-436$.

[42] R. M. Anderson, R. M. May, Infectious Diseases of Humans: Dynamics and Control, Oxford University Press, Oxford, UK, 1991. 
[43] P. C. G. Nunes, R. P. Daumas, J. C. Sánchez-Arcila, R. M. R. Nogueira, M. A. P. Horta, F. B. dos Santos, 30 years of fatal dengue cases in Brazil: a review, BMC Public Health 19 (2019) 329 .

[44] S. B. Maier, Determination of the optimal age to vaccinate against dengue using a tetravalent dengue vaccine in Brazil, Unpublished PhD Thesis, University of Strathclyde (2019).

[45] N. M. Ferguson, I. Rodríguez-Barraquer, I. Dorigatti, L. Mier-y Teran-Romero, D. J. Laydon, D. A. Cummings, Benefits and risks of the sanofi-pasteur dengue vaccine: Modeling optimal deployment, Science 353 (2016) 1033-1036.

[46] R. A. Martínez-Vega, G. Carrasquila, E. Luna, J. Ramos-Castañeda, Ade and dengue vaccination, Vaccine 35 (2017) 3910-3912.

[47] A. Wilder-Smith, K. S. Vannice, J. Hombach, J. Farrar, T. Nolan, Population Perspectives and World Health Organization Recommendations for CYD-TDV Dengue Vaccine, The Journal of Infectious Diseases 214 (2016) 1796-1799. 